\title{
Selection of an Effective Hand Sanitizer to Reduce COVID-19 Effects and Extension of TOPSIS Technique Based on Correlation Coefficient under Neutrosophic Hypersoft Set
}

\author{
Abdul Samad (D), ${ }^{1}$ Rana Muhammad Zulqarnain ${ }^{(D},{ }^{2}$ Emre Sermutlu ${ }^{D},{ }^{3}$ Rifaqat Ali $\left(\mathbb{D},{ }^{4}\right.$ \\ Imran Siddique, ${ }^{5}$ Fahd Jarad $\mathbb{C}^{3,6}$ and Thabet Abdeljawad $\mathbb{C}^{7,8}$ \\ ${ }^{1}$ School of Mathematics, Northwest University Xi'an, Xi'an 710127, China \\ ${ }^{2}$ Department of Mathematics, University of Management and Technology Lahore, Sialkot Campus, Lahore, Pakistan \\ ${ }^{3}$ Department of Mathematics, Cankaya University, Etimesgut, Ankara, Turkey \\ ${ }^{4}$ Department of Mathematics, College of Science and Arts, Muhayil, King Khalid University, 61413 Abha, Saudi Arabia \\ ${ }^{5}$ Department of Mathematics, School of Science, University of Management and Technology, Lahore, Pakistan \\ ${ }^{6}$ Department of Medical Research, China Medical University Hospital, China Medical University, Taichung, Taiwan \\ ${ }^{7}$ Department of Mathematics and General Sciences, Prince Sultan University, Riyadh, Saudi Arabia \\ ${ }^{8}$ Department of Computer Science and Information Engineering, Asia University Taichung, Taichung, Taiwan
}

Correspondence should be addressed to Fahd Jarad; fahd@cankaya.edu.tr and Thabet Abdeljawad; tabdeljawad@psu.edu.sa

Received 19 February 2021; Revised 13 March 2021; Accepted 25 March 2021; Published 4 June 2021

Academic Editor: Ahmed Mostafa Khalil

Copyright (c) 2021 Abdul Samad et al. This is an open access article distributed under the Creative Commons Attribution License, which permits unrestricted use, distribution, and reproduction in any medium, provided the original work is properly cited.

Correlation coefficients are used to tackle many issues that include indistinct as well as blurred information excluding is not able to deal with the general fuzziness along with obscurity of the problems that have various information. The correlation coefficient (CC) between two variables plays an important role in statistics. Likewise, the accuracy of relevance assessment depends on the information in a set of discourses. The data collected for numerous statistical studies is full of exceptions. The concept of the neutrosophic hypersoft set (NHSS) is a parameterized family that deals with the subattributes of the parameters and is a proper extension of the neutrosophic soft set to accurately assess the deficiencies, anxiety, and uncertainty in decision-making. Compared with existing research, NHSS can accommodate more uncertainty, which is the most significant technique for describing fuzzy information in the decision-making process. The core objective of follow-up research is to develop the concept and characteristics of CC and the weighted correlation coefficient (WCC) of NHSS. We also introduced some aggregation operators in the considered environment, which can help us establish a prioritization technique for order preference by similarity to the ideal solution (TOPSIS) based on CC and WCC under NHSS. A decision-making strategy is established to solve multicriteria group decisionmaking (MCGDM) problems utilizing developed methodology. Moreover, the proposed method is utilized for the selection of an effective hand sanitizer during the COVID-19 pandemic to ensure the validity of the proposed approach. The practicality, effectivity, and flexibility of the current approach are proved through comparative analysis with the assistance of some existing studies.

\section{Introduction}

Correlation plays an essential role in statistics and engineering. Employing correlation analysis, the joint relationship of two variables can be employed to assess the interdependency. In addition to utilizing probabilistic methods to such a lot of practical engineering complications, you also can locate several barriers to probabilistic strategies. Incidentally, the possibility of this process relies on a large amount of information, which may be random. However, structures have various uncertainties that are difficult to elevate, and it is difficult to obtain exact outcomes. So, due to unexplainable extensive information, the consequences of probability theory cannot provide experts with suitable 
information. Also, in real-world problems, there are no adequate grounds to proceed with the renowned statistical data. The consequences of probability theory are not in favor of experts because of initial hurdles. So, the probability theory has insufficient competency to resolve the uncertainty stated in the data. Various investigators in the world have predetermined and recommended different methods to solve such issues which involve anxiety. Zadeh suggested the idea of fuzzy sets (FS) [1] to solve complex issues which included anxiety as well as uncertainty. The fuzzy set theory allows modern ratings of the Mem of elements in the set. This is represented by the Mem function, and the effective unit interval of the Mem function is $[0,1]$. The fuzzy set is the generalization of the classical set because the indicator function of the classic set is a special case of the Mem function of the fuzzy set if the latter only takes the value 0 or 1. In the fuzzy set theory, the classical bivalent set is usually called the crisp set. Fuzzy set theory can be used in a wide range of fields with incomplete or imprecise information.

In some circumstances, decision makers consider the Mem and nonmembership (Nmem) values of objects. In such cases, Zadeh's FS is unable to handle the imprecise and vague information. Atanassov [2] developed the notion of intuitionistic fuzzy sets (IFS) to deal the abovementioned difficulties. The IFS accommodates the imprecise and inaccurate information using Mem and Nmem values. Atanassov IFS is unable to solve those problems in which decision makers considered the membership degree (MD) and nonmembership degree (NMD) such as $\mathrm{MD}=0.5$ and $\mathrm{NDM}=0.8$; then, $0.5+0.8 \not 1$. Yager $[3,4]$ extended the notion of IFS to Pythagorean fuzzy sets (PFSs) to overcome the above-discussed complications by modifying $\mathrm{MD}+\mathrm{NMD} \leq 1$ to $\mathrm{MD}^{2}+\mathrm{NMD}^{2} \leq 1$. After the development of PFSs, Zhang and $\mathrm{Xu}$ [5] proposed operational laws for PFSs and established a DM approach to resolving the MCDM problem. Wei and Lu [6] proposed Pythagorean fuzzy power aggregation operators (AOs) and established the DM methodologies to solve multiattribute decisionmaking problems (MADM) using their developed AOs. Wang and Li [7] presented interaction operational laws and power Bonferroni mean operators for PFSs with their basic properties. Gao et al. [8] presented several aggregation operators by considering the interaction and proposed a DM approach to solving MADM difficulties by utilizing the developed operators. Wei [9] developed the interaction operational laws for Pythagorean fuzzy numbers (PFNs) by considering interaction and established interaction aggregation operators by using the developed interaction operations. Zhang [10] developed the accuracy function and presented a DM approach to solve multiple criteria group decision-making (MCGDM) problems using PFNs. Wang et al. [11] extended the PFSs and introduced interactive Hamacher operation with some novel AOs. They also established a DM method to solve MADM problems by using their proposed operators. Wang and Li [12] developed some operators for interval-valued PFSs and utilized their operators to resolve multiattribute group decision-making (MAGDM) problems. Peng and Yuan [13] established some novel operators such as Pythagorean fuzzy point operators and developed DM techniques using their proposed operators. Peng and Yang [14] introduced some operations with their desirable properties under PFSs and planned DM methodology to solve the MAGDM problem. Garg [15] developed the logarithmic operational laws for PFSs and proposed some AOs. Arora and Garg [16] presented the operational laws for linguistic IFS and developed prioritized AOs. Ma and Xu [17] presented some innovative AOs for PFSs, and they also developed the score and accuracy functions for PFNs.

Abovementioned theories and their DM methodologies have been used in several fields of life. However, these theories are unable to deal with the parametrization of the alternatives. Molodtsov [18] developed the soft sets (SS) to overcome the abovementioned complications. Molodtsov's SS competently deals with imprecise, vague, and unclear information of objects considering their parametrization. Maji et al. [19] prolonged the concept of SS and introduced basic operations with their properties. Maji et al. [20] established a DM technique using their developed operations for SS. They also merged two well-known theories such as FS and SS and established the concept of fuzzy soft sets (FSS) [21]. They also proposed the notion of an intuitionistic fuzzy soft set (IFSS) [22] and discussed their basic operations. Garg and Arora [23] extended the notion of IFSS and presented a generalized form of IFSS with AOs. They also planned a DM technique to resolve undefined and inaccurate information under IFSS information. Garg and Arora [24] presented the correlation and weighted correlation coefficients for IFSS and extended the TOPSIS technique using developed correlation measures. Zulqarnain et al. [25] introduced some AOs and correlation coefficients for interval-valued IFSS. They also extended the TOPSIS technique using their developed correlation measures and utilized them to solve the MADM problem. Peng et al. [26] proposed the Pythagorean fuzzy soft sets (PFSSs) and presented fundamental operations of PFSSs with their desirable properties by merging PFS and SS. Athira et al. [27] extended the notion of PFSSs and proposed entropy measures for PFSSs. They also presented some distance measures for PFSSs and utilized their developed distance measures to solve DM [28] issues. Zulqarnain et al. [29] introduced operational laws for Pythagorean fuzzy soft numbers (PFSNs) and developed AOs such as Pythagorean fuzzy soft weighted average and geometric by using defined operational laws for PFSNs. They also planned a DM approach to solve MADM problems with the help of presented operators. Riaz et al. [30] prolonged the idea of PFSSs and developed the $m$ polar PFSSs. They also established the TOPSIS method under-considered hybrid structure and proposed a DM methodology to solve the MCGDM problem. Riaz et al. [31] developed the similarity measures for PFSS with their fundamental properties. Han et al. [32] protracted the TOPSIS method under PFSSs' environment and utilized their developed approach to solving the MAGDM problem. Zulqarnain et al. [33] planned the TOPSIS methodology in the PFSS environment based on the correlation coefficient. They also established a DM methodology to resolve the MCGDM 
concerns and utilized the developed approach in green supply chain management.

All the above studies only deal the insufficient data considering membership and nonmembership values; however, these theories cannot handle the overall incompatible as well as imprecise information. To address such incompatible as well as imprecise records, the idea of the neutrosophic set (NS) was developed by Smarandache [34]. Maji [35] offered the idea of a neutrosophic soft set (NSS) with necessary operations and properties. Karaaslan [36] developed the idea of the possibility NSS and introduced a possibility of neutrosophic soft DM method to solve those problems which contain uncertainty based on And-product. Broumi [37] developed the generalized NSS with some operations and properties and used the projected concept for DM. To solve MCDM problems with single-valued neutrosophic numbers (SVNNs) presented by Deli and Subas in [38], they constructed the concept of cut sets of SVNNs. Based on the correlation of IFS, the term correlation coefficient (CC) of SVNSs [39] was introduced. Ye [40] introduced the simplified NSs with some operational laws and AOs such as weighted arithmetic and weighted geometric average operators and constructed an MCDM method based on his proposed AOs. Masooma et al. [41] progressed a new concept through combining the multipolar fuzzy set and neutrosophic set which is known as the multipolar neutrosophic set, and they also established various characterization and operations with examples. Zulqarnain et al. [42] presented the generalized neutrosophic TOPSIS and used their presented technique for supplier selection in the production industry.

Smarandache [43] protracted the idea of SS to hypersoft sets (HSS) by substituting the one-parameter function $f$ to a multiparameter (subattribute) function. Samarandache claimed that the established HSS is competently dealing with uncertain objects comparative to SS. Nowadays, HSS theory and its extensions have been arising unexpectedly. Several investigators go through progressed distinctive operators along with characteristics under HSS and its extensions $[44,45]$. Zulqarnain et al. [46] presented the IFHSS which is the generalized version of IFSS. They established the TOPSIS method to resolve the MADM problem utilizing the developed correlation coefficient. The authors of [47] introduced the Pythagorean fuzzy hypersoft sets with some basic operations and their properties. They also established a decision-making technique to deal with decision-making complications. Zulqarnain et al. [48] proposed the Pythagorean fuzzy hypersoft sets with AOs and correlation coefficients. They also established the TOPSIS technique using their developed correlation coefficient and utilized the presented approach for the selection of appropriate antivirus face masks. Zulqarnain et al. [49] presented some novel operations for interval-valued Pythagorean fuzzy hypersoft sets and discussed their desirable properties. They also developed the correlation coefficient and weighted correlation coefficient and developed a decision-making technique to solve decision-making issues utilizing their developed correlation measures [50] because the above work is considered to examine the environment of linear inequality between the
MD and NMD of subattributes of the considered attributes. However, all existing studies only deal with the scenario by using MD and NMD of subattributes of the considered attributes. If any decision maker considers the truthiness, falsity, and indeterminacy of any subattribute of the alternatives, then, clearly, we can see that it cannot be handled by the abovementioned theories. To overcome the above limitations, we proposed some AOs for NHSS such as neutrosophic hypersoft weighted average and neutrosophic hypersoft weighted geometric operators. The core objective of the following scientific research is to develop novel AOs for the NHSS environment and processing mechanism, which can also follow the assumptions of NHSNs. Furthermore, the TOPSIS technique to solve the MCGDM problem was developed and a numerical illustration to justify the effectiveness of the proposed approach under the NHSS environment was presented.

The rest of the article can be summarized as follows. In Section 2, we introduced the fundamental notions such as SS, NSS, and NHSS, which can help us to build the subsequent research structure. In Section 3, we planned the correlation and relationship with NHSS informational energies and used the correlation and informational energies to develop CC, WCC, and their characteristics. Also, we use the planned CC to establish the TOPSIS method underconsidered environment and propose some aggregation operators. To solve the MCGDM problem, an algorithm is established by using the presented TOPSIS approach, and a numerical explanation is provided in Section 4. Moreover, the planned DM method is used for the selection of multipurpose hand sanitizer in the COVID-19 pandemic. Also, we apply some available techniques to propose a comparative analysis of our planned approach. Similarly, the benefits of the planned algorithmic rule, superiority, tractability, and effectivity are presented. We will briefly discuss and equate the proposed strategy along with available methodologies in Section 5.

\section{Preliminaries}

In the following section, we recalled fundamental concepts that help us to develop the structure of the current article such as SS, NS, NSS, HSS, FHSS, and NHSS.

Definition 1 (see [18]). Let $\mathscr{U}$ and $\mathscr{E}$ be the universe of discourse and set of attributes, respectively. Let $\mathscr{P}(\mathscr{U})$ be the power set of $\mathscr{U}$ and $\mathscr{A} \subseteq \mathscr{E}$. A pair $(\mathscr{F}, \mathscr{A})$ is called a SS over $\mathscr{U}$, and its mapping is expressed as follows:

$$
\mathscr{F}: \mathscr{A} \longrightarrow \mathscr{P}(\mathscr{U})
$$

Also, it can be defined as follows:

$$
(\mathscr{F}, \mathscr{A})=\{\mathscr{F}(e) \in \mathscr{P}(\mathscr{U}): e \in \mathscr{E}, \mathscr{F}(e)=\varnothing \text { if } e \notin \mathscr{A}\} .
$$

Maji et al. [21] explored the theory of FS and SS and planned a more generalized version to handle the uncertainty compared with the existing FS and SS along with its 
unique features. This is generally known as a fuzzy soft set, which is a combination of FS and SS.

Definition 2 (see [21]). Let $\mathscr{U}$ and $\mathscr{E}$ be a universe of discourse and set of attributes, respectively, and $\mathscr{F}(\mathscr{U})$ be a power set of $\mathscr{U}$. Let $\mathscr{A} \subseteq \mathscr{E}$; then, $(\mathscr{F}, \mathscr{A})$ is an FSS over $\mathcal{U}$, and its mapping can be expressed as follows:

$$
\mathscr{F}: \mathscr{A} \longrightarrow \mathscr{F}(\mathscr{U}) \text {. }
$$

Definition 3 (see [34]). Let $\mathscr{U}$ be a universe and $\mathscr{A}$ be an NS on $\mathscr{U}$ which is defined as $\mathscr{A}=\left\{v,\left(\mathscr{T}_{\mathscr{A}}(v), \mathscr{I}_{\mathscr{A}}(v)\right.\right.$, $\left.\left.\mathfrak{S}_{\mathscr{A}}(v)\right): v \in \mathscr{U}\right\}$, where $\left.\mathscr{T}, \mathscr{I}, \mathfrak{E}: \mathcal{U} \longrightarrow\right] 0^{-}, 1^{+}[$and $0^{-} \leq \mathscr{T}_{\mathscr{A}}(v)+\mathscr{I}_{\mathscr{A}}(v)+\mathfrak{C}_{\mathscr{A}}(v) \leq 3^{+}$.

Maji et al. [35] established the notion of the neutrosophic soft set by merging the two existing theories such as NS and SS with some basic operations and their properties.

Definition 4 (see [35]). Let $\mathcal{U}$ be the universal set and $\mathscr{E}$ be the set of attributes concerning $\mathcal{U}$. Let $\mathscr{P}(\mathcal{U})$ be the set of neutrosophic values of $\mathscr{U}$ and $\mathscr{A} \subseteq \mathscr{E}$. A pair $(\mathscr{F}, \mathscr{A})$ is called a neutrosophic soft set over $\mathcal{U}$ and its mapping is given as

$$
\mathscr{F}: \mathscr{A} \longrightarrow \mathscr{P}(\mathscr{U}) \text {. }
$$

Definition 5 (see [43]). Let $\mathcal{U}$ be a universe of discourse and $\mathscr{P}(\mathscr{U})$ be a power set of $\mathscr{U}$ and $k=\left\{k_{1}, k_{2}, k_{3}, \ldots, k_{n}\right\},(n \geq 1)$ and $K_{i}$ represented the set of attributes and their corresponding subattributes such as $K_{i} \cap K_{j}=\varphi$, where $i \neq j$ for

each $n \geq 1$ and $i, j \in\{1,2,3, \ldots, n\}$. Assume $K_{1} \times K_{2} \times K_{3} \times$ $\ldots \times K_{n}=\stackrel{\dot{\mathrm{t}}}{A}=\left\{a_{1 h} \times a_{2 k} \times \cdots \times a_{n l}\right\}$ is a collection of subattributes, where $1 \leq h \leq \alpha, 1 \leq k \leq \beta$, and $1 \leq l \leq \gamma$, and $\alpha$,

$\beta, \gamma \in \mathbb{N}$. Then, the pair $\left(\mathscr{F}, K_{1} \times K_{2} \times K_{3} \times \ldots \times K_{n}=\stackrel{\dot{t}}{A}\right)$ is known as HSS defined as follows:

$$
\mathscr{F}: K_{1} \times K_{2} \times K_{3} \times \cdots \times K_{n}=\stackrel{\mathfrak{t}}{A} \longrightarrow \mathscr{P}(\mathscr{U}) .
$$

It is also defined as

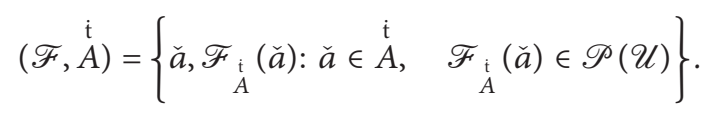

Definition 6 (see [43]). Let $\mathcal{U}$ be a universe of discourse and $\mathscr{P}(\mathcal{U})$ be a power set of $\mathcal{U}$ and $k=\left\{k_{1}, k_{2}, k_{3}, \ldots, k_{n}\right\},(n \geq 1)$ be a set of attributes, and set $K_{i}$ as a set of corresponding subattributes of $k_{i}$, respectively, with $K_{i} \cap K_{j}=\varphi$ for $n \geq 1$ for each $i, j \in\{1,2,3, \ldots, n\}$ and $i \neq j$. Assume $K_{1} \times K_{2} \times K_{3} \times \ldots \times K_{n}=\stackrel{\dot{t}}{\mathscr{A}}=\left\{a_{1 h} \times a_{2 k} \times \cdots \times a_{n l}\right\}$ is a collection of subattributes, where $1 \leq h . . \alpha, 1 \leq k \leq \beta$, and $1 \leq l \leq \gamma$, and $\alpha, \beta$, and $\gamma \in \mathbb{N}$, and IFS $^{\mathscr{U}}$ is a collection of all intuitionistic fuzzy subsets over $\mathcal{U}$. Then, the pair $(\mathscr{F}$, $\left.K_{1} \times K_{2} \times K_{3} \times \ldots \times K_{n}=\stackrel{\dot{A}}{\mathscr{A}}\right)$ is said to be IFHSS over $\mathcal{U}$, and its mapping is defined as

$$
\mathscr{F}: K_{1} \times K_{2} \times K_{3} \times \cdots \times K_{n}=\stackrel{i t}{\mathscr{A}} \longrightarrow \mathrm{IFS}^{\mathscr{U}}
$$

It is also defined as

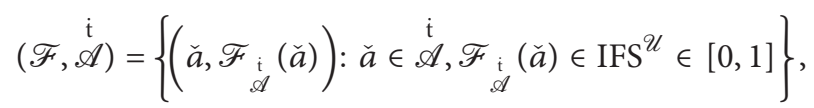

where $\quad \mathscr{F}_{\mathrm{t}}(\check{a})=\left\{\delta, \sigma_{\mathscr{F}(\check{a})}(\delta), \tau_{\mathscr{F}(\check{a})}(\delta): \delta \in \mathcal{U}\right\}$, where $\sigma_{\mathscr{F}(\check{a})}(\delta)$ and $\tau_{\mathscr{F}(\breve{a})}(\delta)$ represent the membership and nonmembership values of the attributes such as $\sigma_{\mathscr{F}(\breve{a})}(\delta)$, $\tau_{\mathscr{F}(\breve{a})}(\delta) \in[0,1]$ and $0 \leq \sigma_{\mathscr{F}(\breve{a})}(\delta)+\tau_{\mathscr{F}(\breve{a})}(\delta) \leq 1$.

Simply an intuitionistic fuzzy hypersoft number (IFHSN) can be expressed as $\mathscr{F}=\left\{\left(\sigma_{\mathscr{F}(\breve{a})}(\delta), \tau_{\mathscr{F}(\check{a})}(\delta)\right)\right\}$, where $0 \leq \sigma_{\mathscr{F}(\breve{a})}(\delta)+\tau_{\mathscr{F}(\check{a})}(\delta) \leq 1$.

The abovementioned IFHSS only deals with the MD and NMD of subattributes, and it is unable to accommodate the indeterminacy of the multi-subattributes of the considered attributes. To overcome such complications, the concept of NHSS has been developed by Smarandache.

Definition 7 (see [43]). Let $\mathcal{U}$ be a universe of discourse and $\mathscr{P}(\mathcal{U})$ be a power set of $\mathcal{U}$ and $k=\left\{k_{1}, k_{2}, k_{3}, \ldots, k_{n}\right\},(n \geq 1)$, and $K_{i}$ represented the set of attributes and their corresponding subattributes such as $K_{i} \cap K_{j}=\varphi$, where $i \neq j$ for each $n \geq 1$ and $i, j \in\{1,2,3, \ldots, n\}$. Assume $K_{1} \times K_{2} \times K_{3} \times$ $\ldots \times K_{n}=\stackrel{\dot{t}}{A}=\left\{a_{1 h} \times a_{2 k} \times \cdots \times a_{n l}\right\}$ is a collection of subattributes, where $1 \leq h \leq \alpha, 1 \leq k \leq \beta$, and $1 \leq l \leq \gamma$, and $\alpha$, $\beta, \gamma \in \mathbb{N}$, and $\mathrm{NS}^{\mathscr{U}}$ represents neutrosophic subsets over $\mathcal{U}$. Then, $\left(\mathscr{F}, K_{1} \times K_{2} \times K_{3} \times \cdots \times K_{n}=\stackrel{\dot{\mathrm{t}}}{A}\right)$ is called NHSS, and its mapping can be expressed as follows:

$$
\mathscr{F}: K_{1} \times K_{2} \times K_{3} \times \cdots \times K_{n}=\stackrel{\text { t }}{\mathrm{A}} \longrightarrow \mathrm{NS}^{\mathscr{U}} .
$$

It is also defined as $(\mathscr{F}, \stackrel{\grave{\mathrm{t}}}{A})=\left\{\left(\check{a}, \mathscr{F}_{\mathrm{t}}(\check{a})\right): \check{a} \in \stackrel{\dot{\mathrm{t}}}{A}\right.$, $\left.\mathscr{F}_{\mathrm{t}}(\check{a}) \in \mathrm{NS}^{\mathscr{U}}\right\}$, where $\mathscr{F}_{\mathrm{t}}(\check{a})=\left\{v, \mathscr{T}_{\mathscr{F}(\check{a})}(v)^{A}, \mathscr{I}_{\mathscr{F}(\check{a})}(v)\right.$, $\left.\mathfrak{G}_{\mathscr{F}(\breve{a})}^{A}(v): v \in \mathcal{U}\right\}$, where $\mathscr{T}_{\mathscr{F}(\breve{a})}^{\mathrm{A}}(v), \mathscr{I}_{\mathscr{F}(\breve{a})}(v)$, and $\boldsymbol{夭}_{\mathscr{F}(\breve{a})}(v)$ represent the truth, indeterminacy, and falsity grades of the attributes such as $\mathscr{T}_{\mathscr{F}(\check{a})}(v), \mathscr{I}_{\mathscr{F}(\check{a})}(v), \mathfrak{E}_{\mathscr{F}(\check{a})}(v) \in \in[0,1]$, and $0 \leq \mathscr{T}_{\mathscr{F}(\breve{a})}(v)+\mathscr{I}_{\mathscr{F}(\breve{a})}(v)+\mathfrak{C}_{\mathscr{F}(\breve{a})}(v) \leq 3$.

Simply a neutrosophic hypersoft number (NHSN) can be expressed as $\mathscr{F}=\left\{\left(\mathscr{T}_{\mathscr{F}(\breve{a})}(v), \mathscr{I}_{\mathscr{F}(\breve{a})}(v), \mathfrak{C}_{\mathscr{F}(\breve{a})}(v)\right)\right\}$, where $0 \leq \mathscr{T}_{\mathscr{F}(\breve{a})}(v)+\mathscr{I}_{\mathscr{F}(\breve{a})}(v)+\mathfrak{C}_{\mathscr{F}(\breve{a})}(v) \leq 3$.

Example 1. Consider $\mathcal{U}=\left\{v_{1}, v_{2}\right\}$ is a universe of discourse and $\quad \mathfrak{L}=\left\{L_{1}=\right.$ Teaching methdology, $L_{2}=$ Subjects, $L_{3}=$ Classes $\}$ are the considered attributes, and their corresponding $n$-tuple subattributes are given as follows: $L_{1}=\left\{a_{11}=\right.$ project base, $a_{12}=$ class discussion $\}, L_{2}=\left\{a_{21}=\right.$ Mathematics, $a_{22}=$ Computer Science, $a_{23}=$ Statistics $\}$, and Classes $=L_{3}=\left\{a_{31}=\right.$ Masters, $a_{32}=$ Doctorol $\}$. Let $\stackrel{\dot{\mathrm{t}}}{A}=L_{1} \times$ $L_{2} \times L_{3}$ be a set of attributes 


$$
\begin{aligned}
\stackrel{\mathfrak{t}}{A} & =L_{1} \times L_{2} \times L_{3}=\left\{a_{11}, a_{12}\right\} \times\left\{a_{21}, a_{22}, a_{23}\right\} \times\left\{a_{31}, a_{32}\right\} \\
& =\left\{\begin{array}{l}
\left(a_{11}, a_{21}, a_{31}\right),\left(a_{11}, a_{21}, a_{32}\right),\left(a_{11}, a_{22}, a_{31}\right),\left(a_{11}, a_{22}, a_{32}\right),\left(a_{11}, a_{23}, a_{31}\right),\left(a_{11}, a_{23}, a_{32}\right), \\
\left(a_{12}, a_{21}, a_{31}\right),\left(a_{12}, a_{21}, a_{32}\right),\left(a_{12}, a_{22}, a_{31}\right),\left(a_{12}, a_{22}, a_{32}\right),\left(a_{12}, a_{23}, a_{31}\right),\left(a_{12}, a_{23}, a_{32}\right),
\end{array}\right\}, \\
\stackrel{\mathfrak{t}}{A} & =\left\{\check{a}_{1}, \check{a}_{2}, \check{a}_{3}, \check{a}_{4}, \check{a}_{5}, \check{a}_{6}, \check{a}_{7}, \check{a}_{8}, \check{a}_{9}, \check{a}_{10}, \check{a}_{11}, \check{a}_{12}\right\} .
\end{aligned}
$$

Then, the NHSS over $\mathcal{U}$ is given as follows:

$$
(\mathscr{F}, \stackrel{\dot{t}}{A})=\left\{\begin{aligned}
&\left(\breve{a}_{1},\left(\delta_{1},(.6, .3, .8)\right),\left(\delta_{2},(.9, .3, .5)\right)\right),\left(\breve{a}_{2},\left(\delta_{1},(.5, .2, .7)\right),\left(\delta_{2},(.7, .1, .5)\right)\right),\left(\breve{a}_{3},\left(\delta_{1},(.5, .2, .8)\right),\left(\delta_{2},(.4, .3, .4)\right)\right), \\
&\left(\breve{a}_{4},\left(\delta_{1},(.2, .5, .6)\right),\left(\delta_{2},(.5, .1, .6)\right)\right),\left(\breve{a}_{5},\left(\delta_{1},(.8, .4, .3)\right),\left(\delta_{2},(.2, .3, .5)\right)\right),\left(\breve{a}_{6},\left(\delta_{1},(.9, .6, .4)\right),\left(\delta_{2},(.7, .6, .8)\right)\right), \\
&\left(\breve{a}_{7},\left(\delta_{1},(.6, .5, .3)\right),\left(\delta_{2},(.4, .2, .8)\right)\right),\left(\breve{a}_{8},\left(\delta_{1},(.8, .2, .5)\right),\left(\delta_{2},(.6, .8, .4)\right)\right),\left(\breve{a}_{9},\left(\delta_{1},(.7, .4, .9)\right),\left(\delta_{2},(.7, .3, .5)\right)\right), \\
&\left(\breve{a}_{10},\left(\delta_{1},(.8, .4, .6)\right),\left(\delta_{2},(.7, .2, .9)\right)\right),\left(\breve{a}_{11},\left(\delta_{1},(.8, .4, .5)\right),\left(\delta_{2},(.4, .2, .5)\right)\right),\left(\breve{a}_{5},\left(\delta_{1},(.7, .5, .8)\right),\left(\delta_{2},(.7, .5, .9)\right)\right)
\end{aligned}\right\} .
$$

For simplicity, we will express $\mathscr{F}_{v_{i}}\left(\check{a}_{j}\right)=$ $\left\{\left\{\left(\mathscr{T}_{\mathscr{F}(\breve{a})}\left(v_{i}\right), \mathscr{J}_{\mathscr{F}(\breve{a})}\left(v_{i}\right), \mathfrak{夭}_{\mathscr{F}(\breve{a})}\left(v_{i}\right)\right)\right\} \mid v_{i} \in \mathscr{U}\right\} \quad$ as $\quad \mathfrak{J}_{\check{a}_{i j}}=$ $\left\langle\mathscr{T}_{\mathscr{F}\left(\check{a}_{i j}\right)}, \mathscr{J}_{\mathscr{F}\left(\check{a}_{i j}\right)}, \mathfrak{C}_{\mathscr{F}\left(\check{a}_{i j}\right)}\right\rangle$ which is called NHSN, where $0 \leq \mathscr{T}_{\mathscr{F}\left(a_{i j}\right)}+\mathscr{J}_{\mathscr{F}\left(\check{a}_{i j}\right)}+\mathfrak{C}_{\mathscr{F}\left(a_{i j}\right)} \leq 3$ and $\mathscr{T}_{\mathscr{F}\left(\check{a}_{i j}\right)}, \mathscr{F}_{\mathscr{F}\left(\check{a}_{i j}\right)}$, $\mathfrak{S}_{\mathscr{F}\left(\breve{a}_{i j}\right)} \in[0,1]$. The score function for NHSNs $\mathfrak{\Im}_{\check{a}_{i j}}$ is defined as follows:

$$
\delta\left(\widetilde{J}_{\check{a}_{i j}}\right)=\mathscr{T}_{\mathscr{F}\left(\check{a}_{i j}\right)}-\mathfrak{C}_{\mathscr{F}\left(\check{a}_{i j}\right)},
$$

where $\mathcal{S}\left(\mathfrak{J}_{\check{a}_{i j}}\right) \in[-1,1]$, and sometimes the score function is unable to compare any two NHSNs. Like $\mathfrak{J}_{\check{a}_{11}}=.6, .2, .2$ and $\mathfrak{J}_{\check{a}_{12}}=.5, .1, .1$, in such cases, it is difficult to choose which alternative is more appropriate. To handle such types of information, we need to introduce the accuracy function for NHSNs such as follows:

$$
H\left(\mathfrak{J}_{\check{d}_{i j}}\right)=\mathscr{T}_{\mathscr{F}\left(\check{a}_{i j}\right)}+\mathscr{J}_{\mathscr{F}\left(\check{a}_{i j}\right)}+\mathfrak{C}_{\mathscr{F}\left(\check{a}_{i j}\right)}, H\left(\mathfrak{J}_{\check{a}_{i j}}\right) \in[0,1] .
$$

In the following, we present the comparison laws to compare NHSNs $\mathfrak{J}_{\check{a}_{i j}}$ and $\mathfrak{I}_{\check{a}_{i j}}$ such as

(i) If $\mathcal{S}\left(\mathfrak{J}_{\breve{a}_{i j}}\right)>\mathcal{S}\left(\mathfrak{I}_{\breve{a}_{i j}}\right)$, then $\mathfrak{J}_{\breve{a}_{i j}}>\mathfrak{I}_{\check{a}_{i j}}$.

(ii) If $\mathcal{S}\left(\mathfrak{J}_{\check{a}_{i j}}\right)=\mathcal{S}\left(\mathfrak{T}_{\check{a}_{i j}}\right)$, then

(1) If $H\left(\mathfrak{J}_{\check{a}_{i j}}\right)>H\left(\mathfrak{T}_{\check{a}_{i j}}\right)$, then $\mathfrak{J}_{\check{a}_{i j}}>\mathfrak{T}_{\check{a}_{i j}}$

(2) If $H\left(\mathfrak{J}_{\tilde{a}_{i j}}\right)=H\left(\mathfrak{I}_{\check{a}_{i j}}\right)$, then $\mathfrak{J}_{\check{a}_{i j}}=\mathfrak{I}_{\check{a}_{i j}}$.

\section{Correlation Coefficient for Neutrosophic Hypersoft Set}

In the subsequent section, we are going to present the notions of CC and WCC with their necessary properties over NHSS. Also, some aggregation operators for NHSNs will be introduced.

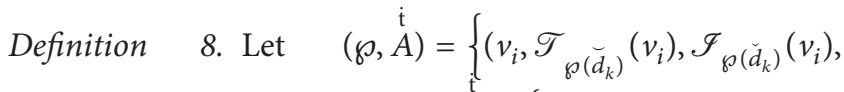
$\left.\left.C_{\mathfrak{\wp}\left(\check{d}_{k}\right)}\left(v_{i}\right)\right) \mid v_{i} \in \mathscr{U}\right\} \quad$ and $\quad(\mathscr{Q}, \stackrel{t}{B})=\left\{\left(v_{i}, \mathscr{T}_{\mathscr{Q}\left(\check{d}_{k}\right)}\left(v_{i}\right), \mathscr{I}_{\mathscr{Q}\left(\check{d}_{k}\right)}\right.\right.$ $\left.\left.\left(v_{i}\right), \mathfrak{C}_{\mathscr{Q}\left(\check{d}_{k}\right)}\left(v_{i}\right)\right) \mid v_{i} \in \mathscr{U}\right\}$ be two NHSSs. Then, their informational neutrosophic energies can be defined as

$$
\begin{aligned}
& \varsigma_{\mathrm{NHSS}}(\mathfrak{\wp}, \stackrel{\grave{\mathrm{t}}}{\mathrm{A}})=\sum_{k=1}^{m} \sum_{i=1}^{n}\left(\left(\mathscr{T}_{\mathfrak{\wp}\left(\check{d}_{k}\right)}\left(v_{i}\right)\right)^{2}+\left(\mathscr{F}_{\mathfrak{\wp}\left(\check{d}_{k}\right)}\left(v_{i}\right)\right)^{2}+\left(\mathfrak{夭}_{\mathfrak{\wp}\left(\check{d}_{k}\right)}\left(v_{i}\right)\right)^{2}\right), \\
& \varsigma_{\text {NHSS }}(\mathscr{Q}, \stackrel{\dot{t}}{\mathrm{~B}})=\sum_{\mathbf{k}=1}^{\mathbf{m}} \sum_{\mathbf{i}=1}^{\mathbf{n}}\left(\left(\mathscr{T}_{\mathscr{Q}\left(\check{d}_{\mathbf{k}}\right)}\left(\mathbf{v}_{\mathbf{i}}\right)\right)^{2}+\left(\mathscr{I}_{\mathscr{Q}\left(\check{d}_{\mathbf{k}}\right)}\left(\mathbf{v}_{\mathbf{i}}\right)\right)^{2}+\left(\mathfrak{c}_{\mathscr{Q}\left(\check{d}_{\mathbf{k}}\right)}\left(\mathbf{v}_{\mathbf{i}}\right)\right)^{2}\right) \text {. }
\end{aligned}
$$

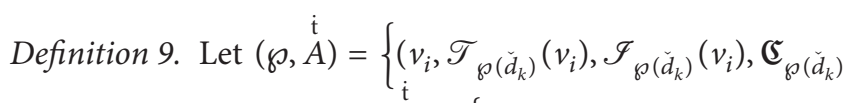
$\left.\left.\left(v_{i}\right)\right) \mid v_{i} \in \mathscr{U}\right\} \quad$ and $\quad(\mathscr{Q}, B)=\left\{\left(v_{i}, \mathscr{T}_{\mathscr{Q}\left(\check{d}_{k}\right)}\left(v_{i}\right), \mathscr{I}_{\mathscr{Q}\left(\check{d}_{k}\right)}\left(v_{i}\right)\right.\right.$,
$\left.\left.\mathfrak{C}_{Q}\left(\check{d}_{k}\right)\left(v_{i}\right)\right) \mid v_{i} \in \mathcal{U}\right\}$ be two NHSSs. Then, the correlation among them can be defined as

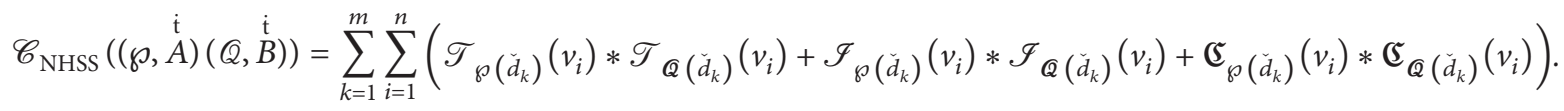


Proposition 1. Let $(\wp, \stackrel{\dot{t}}{A})=\left\{\left(v_{i}, \mathscr{T}_{\wp\left(\check{d}_{k}\right)}\left(v_{i}\right), \mathscr{I}_{\mathfrak{f}\left(\check{d}_{k}\right)} \quad\left(v_{i}\right)\right.\right.$, $\left.\left.\mathfrak{C}_{\wp\left(\check{d}_{k}\right)}\left(v_{i}\right)\right) \mid v_{i} \in \mathscr{U}\right\}$ and $(\mathscr{Q}, B)=\left\{\left(v_{i}, \mathscr{T}_{\mathscr{Q}\left(\check{d}_{k}\right)}\left(v_{i}\right), \mathscr{I}_{\mathscr{Q}\left(\check{d}_{k}\right)}\right.\right.$ $\left.\left.\left(v_{i}\right), \mathfrak{夭}_{\mathscr{Q}\left(\check{l}_{k}\right)}\left(v_{i}\right)\right) \mid v_{i} \in \mathscr{U}\right\}$ be two NHSSs and $\mathscr{C}_{N H S S}((\wp, \stackrel{\dot{t}}{A})$, $(\mathbb{Q}, \stackrel{t}{B}))$ be a correlation between them and satisfied the following properties:

(1) $\mathscr{C}_{\text {NHSS }}((\wp, \stackrel{\dot{t}}{A}),(\wp, \stackrel{\dot{t}}{A}))=\varsigma_{\text {NHSS }}(\wp, \stackrel{\dot{t}}{A})$

(2) $\mathscr{C}_{N H S S}((\mathbb{Q}, \stackrel{t}{B}),(\mathscr{Q}, \stackrel{t}{B}))=\varsigma_{N H S S}(\mathbb{Q}, B)$
Proof. By utilizing equation (15), it easily can be proved.

Definition 10. Let $\quad(\wp, \stackrel{\dot{t}}{A})=\left\{\left(v_{i}, \mathscr{T}_{\mathscr{\wp}\left(\check{d}_{k}\right)}\left(v_{i}\right), \mathscr{I}_{\wp\left(\check{d}_{k}\right)}\left(v_{i}\right)\right.\right.$, $\left.\left.\mathfrak{G}_{\mathfrak{f}\left(\check{d}_{k}\right)}\left(v_{i}\right)\right) \mid v_{i} \in \mathscr{U}\right\}$ and $(\mathscr{Q}, B)=\left\{\left(v_{i}, \mathscr{T}_{\mathscr{Q}\left(\check{d}_{k}\right)}\left(v_{i}\right), \mathscr{I}_{\mathscr{Q}\left(\check{d}_{k}\right)}\left(v_{i}\right)\right.\right.$, $\left.\left.\mathfrak{C}_{\mathscr{Q}\left(\breve{d}_{k}\right)}\left(v_{i}\right)\right) \mid v_{i} \in \mathscr{U}\right\}$ be two NHSSs; then, CC between them is given as $\delta_{\text {NHSS }}((\wp, \stackrel{\mathfrak{t}}{A}),(\mathscr{Q}, \stackrel{\mathfrak{t}}{\mathrm{B}}))$ and expressed as follows:

$\delta_{\text {NHSS }}((\wp, \stackrel{\mathfrak{t}}{A}),(\mathscr{Q}, \stackrel{\mathfrak{t}}{B}))=\frac{\mathscr{C}_{\text {NHSS }}((\wp, \stackrel{\mathfrak{t}}{A}),(\mathscr{Q}, \stackrel{\mathfrak{t}}{B}))}{\sqrt{\wp_{\text {NHSS }}(\wp, \stackrel{\mathfrak{t}}{A})} * \sqrt{\wp_{\text {NHSS }}(\mathscr{Q}, \stackrel{\mathfrak{t}}{B})}}$,

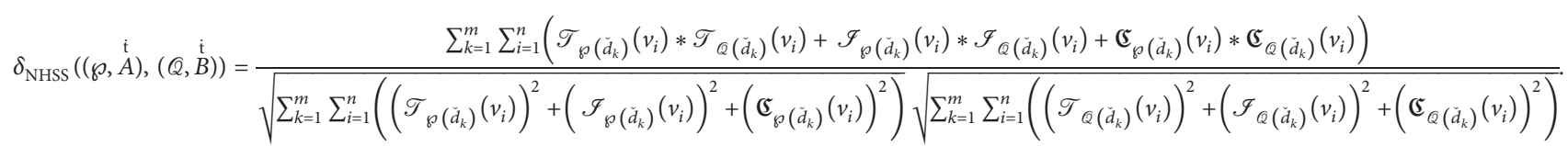

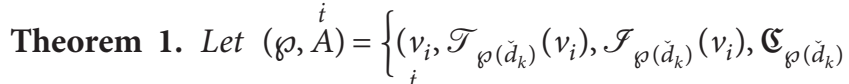
$\left.\left.\left(v_{i}\right)\right) \mid v_{i} \in \mathscr{U}\right\} \quad$ and $\quad(\mathscr{Q}, B)=\left\{\left(v_{i}, \mathscr{T}_{\mathscr{Q}\left(\check{d}_{k}\right)}\left(v_{i}\right), \mathscr{I}_{\mathscr{Q}\left(\check{d}_{k}\right)}\left(v_{i}\right)\right.\right.$, $\left.\left.\mathfrak{C}_{Q}\left(\check{d}_{k}\right)\left(v_{i}\right)\right) \mid v_{i} \in \mathscr{U}\right\}$ be two NHSSs; then, CC between them satisfies the following properties:

(1) $0 \leq \delta_{N H S S}((\bullet, \stackrel{t}{A}),(\stackrel{i}{t}, \stackrel{t}{B})) \leq 1$

(2) $\delta_{\text {NHSS }}((\wp, A),(\mathscr{Q}, \stackrel{t}{B}))=\delta_{\text {NHSS }}((\mathscr{Q}, \stackrel{i}{B}),(\wp, \stackrel{t}{A}))$
(3) If $(\wp, \stackrel{\dot{t}}{A})=(\mathscr{Q}, \stackrel{\dot{Q}}{B})$, that is, $\forall i, k, \mathfrak{I}_{\wp\left(\check{d}_{k}\right)}\left(v_{i}\right)=$ $\mathfrak{I}_{\mathscr{Q}\left(\check{d}_{k}\right)}\left(v_{i}\right), \mathscr{I}_{\mathscr{Q}\left(\check{d}_{k}\right)}\left(v_{i}\right)=\mathscr{I}_{\mathscr{Q}\left(\check{d}_{k}\right)}\left(v_{i}\right)$, and $\mathfrak{C}_{\mathscr{f}\left(\check{d}_{k}\right)}\left(v_{i}\right)=$ $\mathfrak{C}_{\mathbb{Q}\left(\check{d}_{k}\right)}\left(v_{i}\right)$, then $\delta_{\text {NHSS }}((\wp, \stackrel{t}{A}),(\mathscr{Q}, \stackrel{\dot{t}}{B}))=1$

Proof. $\quad \delta_{\text {NHSS }}((\wp, \stackrel{\mathfrak{t}}{A}),(\mathscr{Q}, \stackrel{\mathfrak{t}}{B})) \geq 0$ is trivial; here, we only need to prove that $\delta_{\text {NHSS }}((\wp, A),(\mathscr{Q}, B)) \leq 1$.

From equation (15), we have

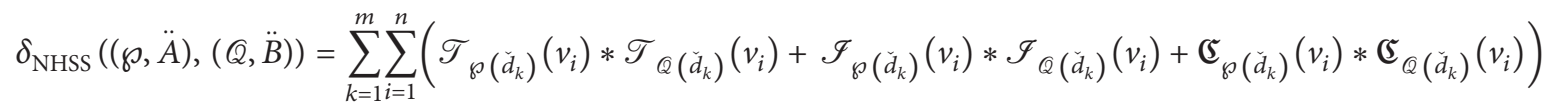

$$
\begin{aligned}
& =\sum_{k=1}^{m}\left(\mathscr{T}_{\mathfrak{Q}\left(\check{d}_{k}\right)}\left(v_{1}\right) * \mathscr{T}_{\mathscr{Q}\left(\check{d}_{k}\right)}\left(v_{1}\right)+\mathscr{I}_{\mathfrak{\wp}\left(\check{d}_{k}\right)}\left(v_{1}\right) * \mathscr{I}_{\mathscr{Q}\left(\check{d}_{k}\right)}\left(v_{1}\right)+\mathfrak{C}_{\mathfrak{O}\left(\check{d}_{k}\right)}\left(v_{1}\right) * \mathfrak{C}_{\mathscr{Q}\left(\check{d}_{k}\right)}\left(v_{1}\right)\right) \\
& +\sum_{k=1}^{m}\left(\mathscr{T}_{\mathfrak{Q}\left(\check{d}_{k}\right)}\left(v_{2}\right) * \mathscr{T}_{\mathscr{Q}\left(\check{d}_{k}\right)}\left(v_{2}\right)+\mathscr{I}_{\mathfrak{\wp}\left(\check{d}_{k}\right)}\left(v_{2}\right) * \mathscr{I}_{\mathscr{Q}\left(\check{d}_{k}\right)}\left(v_{2}\right)+\mathfrak{C}_{\mathfrak{\wp}\left(\check{d}_{k}\right)}\left(v_{2}\right) * \mathfrak{C}_{\mathscr{Q}\left(\check{d}_{k}\right)}\left(v_{2}\right)\right) \\
& + \\
& \text { : } \\
& + \\
& \sum_{k=1}^{m}\left(\mathscr{T}_{\mathfrak{\wp}\left(\check{d}_{k}\right)}\left(v_{n}\right) * \mathscr{T}_{\mathscr{Q}\left(\check{d}_{k}\right)}\left(v_{n}\right)+\mathscr{I}_{\mathfrak{\wp}\left(\check{d}_{k}\right)}\left(v_{n}\right) * \mathscr{I}_{\mathscr{Q}\left(\check{d}_{k}\right)}\left(v_{n}\right)+\mathfrak{C}_{\mathfrak{\wp}\left(\check{d}_{k}\right)}\left(v_{n}\right) * \mathfrak{C}_{\mathbb{Q}\left(\check{d}_{k}\right)}\left(v_{n}\right)\right), \\
& \delta_{\mathrm{NHSS}}((\wp, \ddot{A}),(\mathbb{Q}, \ddot{B}))
\end{aligned}
$$

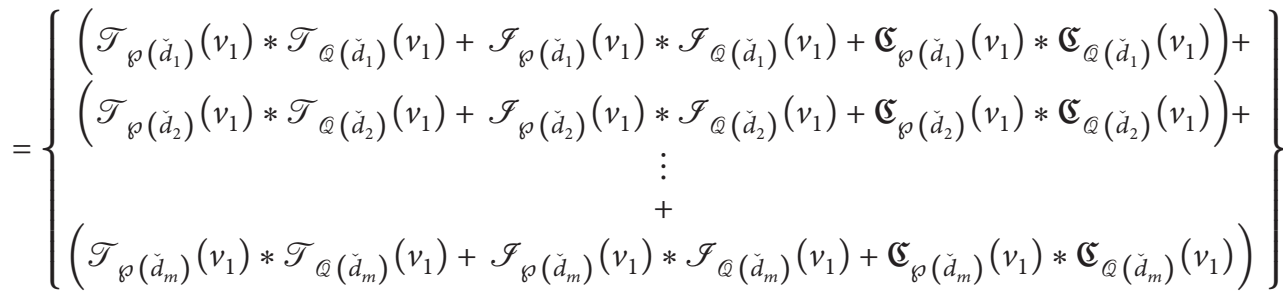




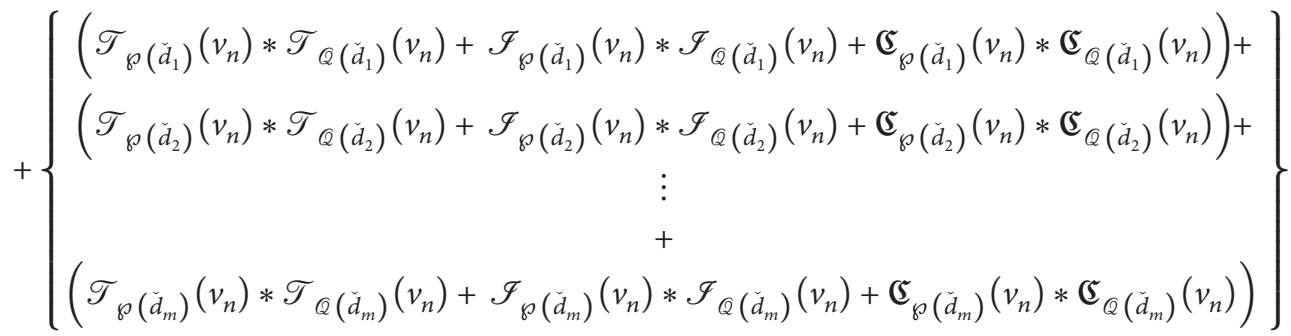

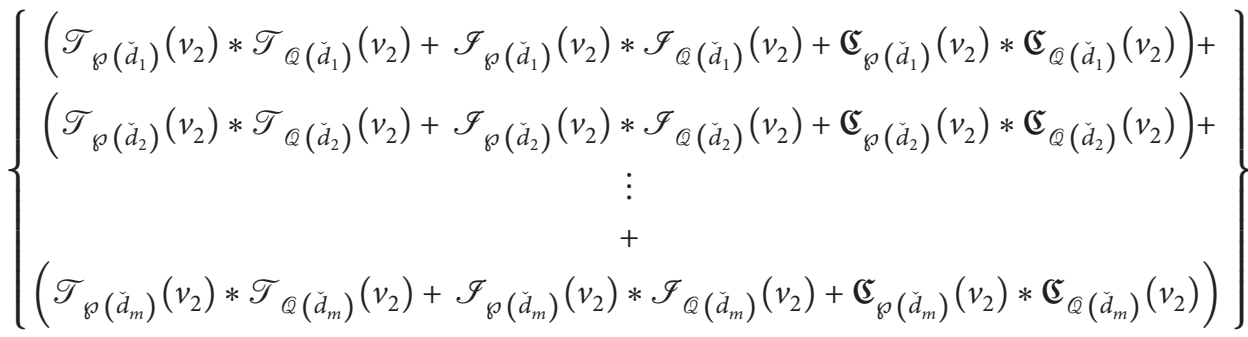

$$
\begin{aligned}
& \sum_{k=1}^{m}\left(\left(\mathscr{T}_{\mathfrak{\wp}\left(\check{d}_{k}\right)}\left(v_{1}\right) * \mathscr{T}_{\mathscr{Q}\left(\check{d}_{k}\right)}\left(v_{1}\right)\right)+\left(\mathscr{T}_{\mathfrak{Q}\left(\check{d}_{k}\right)}\left(v_{2}\right) * \mathscr{T}_{\mathscr{Q}\left(\check{d}_{k}\right)}\left(v_{2}\right)\right)+\cdots+\left(\mathscr{T}_{\mathfrak{Q}\left(\check{d}_{k}\right)}\left(v_{n}\right) * \mathscr{T}_{\mathscr{Q}\left(\check{d}_{k}\right)}\left(v_{n}\right)\right)\right)+ \\
& \sum_{k=1}^{m}\left(\left(\mathscr{I}_{\mathscr{\wp}\left(\check{d}_{k}\right)}\left(v_{1}\right) * \mathscr{I}_{\mathscr{Q}\left(\check{d}_{k}\right)}\left(v_{1}\right)\right)+\left(\mathscr{I}_{\mathscr{O}\left(\check{d}_{k}\right)}\left(v_{2}\right) * \mathscr{I}_{\mathscr{Q}\left(\check{d}_{k}\right)}\left(v_{2}\right)\right)+\cdots+\left(\mathscr{I}_{\mathfrak{f}\left(\check{d}_{k}\right)}\left(v_{n}\right) * \mathscr{I}_{\mathbb{Q}\left(\check{d}_{k}\right)}\left(v_{n}\right)\right)\right)+ \\
& \sum_{k=1}^{m}\left(\left(\mathfrak{夭}_{\wp\left(\check{d}_{k}\right)}\left(v_{1}\right) * \mathfrak{\complement}_{\mathscr{Q}\left(\check{d}_{k}\right)}\left(v_{1}\right)\right)+\left(\mathfrak{夭}_{\wp\left(\check{d}_{k}\right)}\left(v_{2}\right) * \mathfrak{C}_{\mathbb{Q}\left(\check{d}_{k}\right)}\left(v_{2}\right)\right)+\cdots+\left(\mathfrak{夭}_{\wp\left(\check{d}_{k}\right)}\left(v_{n}\right) * \mathfrak{C}_{\mathscr{Q}\left(\check{d}_{k}\right)}\left(v_{n}\right)\right)\right) .
\end{aligned}
$$

By using Cauchy-Schwarz inequality,

$\delta_{\mathrm{NHSS}}((\wp, \ddot{A}),(Q, \ddot{B}))^{2} \leq$

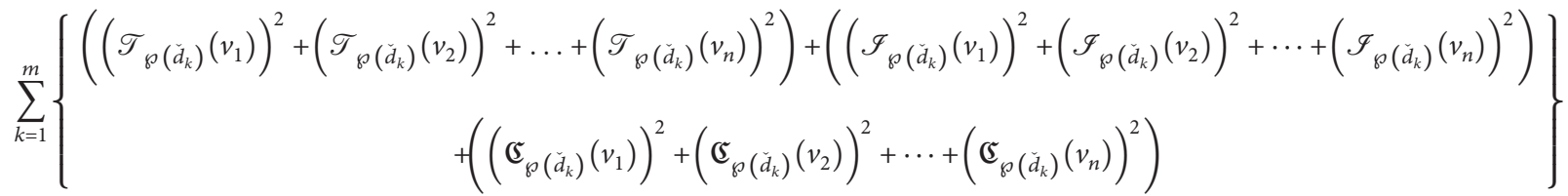

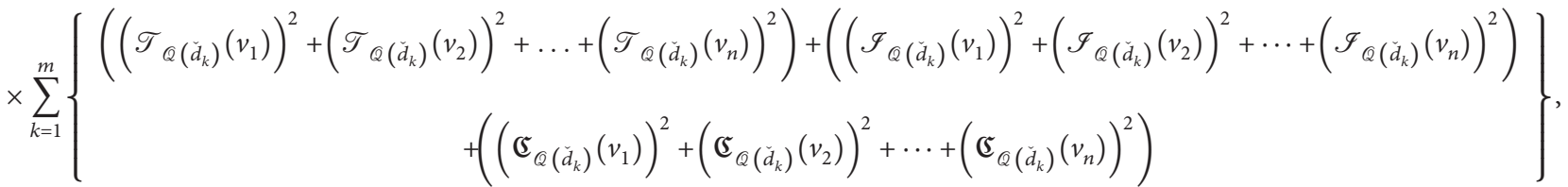

$\delta_{\text {NHSS }}\left((\wp, \stackrel{\mathfrak{t}}{A}),((\mathscr{Q}, \stackrel{\mathfrak{t}}{B}))^{2} \leq\right.$

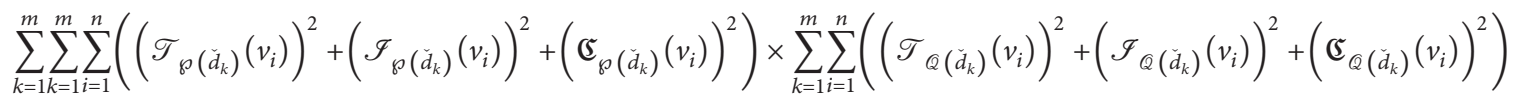

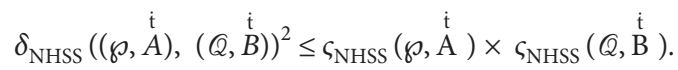




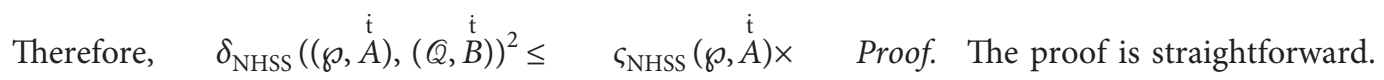
$\varsigma_{\text {NHSS }}(\mathcal{Q}, \stackrel{\dot{\mathfrak{t}}}{B})$. Hence, by using Definition 9 , we have $\delta_{\text {NHSS }}((\wp, \stackrel{\dot{\mathrm{t}}}{A}),(\stackrel{\dot{\mathrm{t}}}{\mathrm{Q}})) \leq 1 . \quad$ So, $\quad 0 \leq \delta_{\mathrm{NHSS}} \quad((\wp, \stackrel{\dot{\mathrm{t}}}{A})$,

Proof. From equation (17), we have $(Q \stackrel{\dot{\mathrm{t}}}{\mathrm{Q}})) \leq 1$.

$\delta_{\text {NHSS }}((\wp, \stackrel{\dot{t}}{A}),(\mathcal{Q}, \stackrel{\stackrel{t}{B}}{)})$

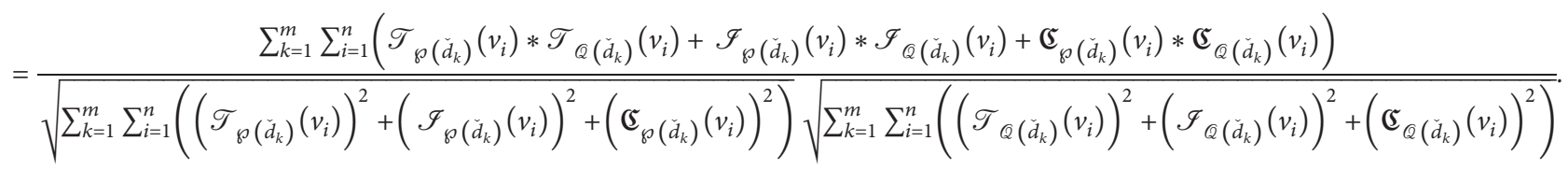

As we know that $\mathscr{T}_{\wp\left(\check{d}_{k}\right)}\left(v_{i}\right)=\mathscr{T}_{\mathscr{Q}\left(\check{d}_{k}\right)}\left(v_{i}\right), \mathscr{I}_{\wp\left(\check{d}_{k}\right)}\left(v_{i}\right)=$ $\mathscr{I}_{Q\left(\check{d}_{k}\right)}\left(v_{i}\right)$, and $\mathfrak{S}_{\wp\left(\check{d}_{k}\right)}\left(v_{i}\right) \stackrel{\mathfrak{C}_{Q}\left(\check{d}_{k}\right)}{ }\left(v_{i}\right) \stackrel{\forall}{\forall} i, k$, we obtain

$\delta_{\text {NHSS }}((\wp, \stackrel{\dot{t}}{A}),(\mathcal{Q}, \stackrel{\dot{t}}{B}))$

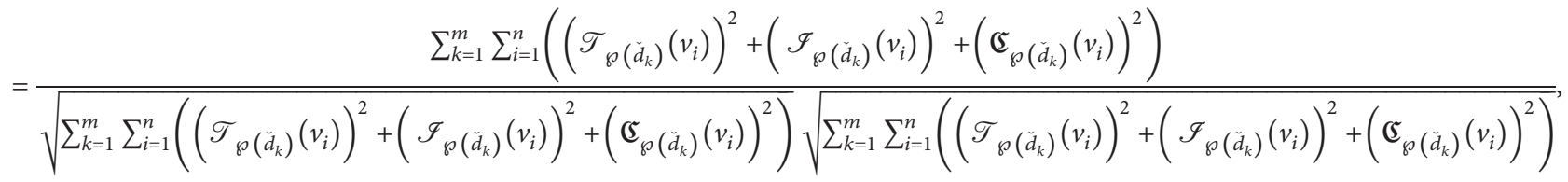

$\delta_{\mathrm{NHSS}}((\wp, \stackrel{\stackrel{\mathrm{t}}{A}}{A}),(\mathcal{Q}, \stackrel{\mathfrak{t}}{B}))=1$

Thus, prove the required result. $\left.\left.\mathfrak{C}_{\mathscr{Q}\left(\breve{d}_{k}\right)}\left(v_{i}\right)\right) \mid v_{i} \in \mathcal{U}\right\}$ be two NHSSs. Then, their correlation

Definition 11 . Let $(\wp, \stackrel{\dot{\mathfrak{t}}}{A})=\left\{\left(v_{i}, \mathscr{T}_{\wp\left(\check{d}_{k}\right)}\left(v_{i}\right), \mathscr{I}_{\wp\left(\check{d}_{k}\right)}\left(v_{i}\right)\right.\right.$, $\left.\left.\mathfrak{S}_{\mathscr{\wp}\left(\check{d}_{k}\right)}\left(v_{i}\right)\right) \mid v_{i} \in \mathscr{U}\right\}$ and $(\mathscr{Q}, \stackrel{\dot{t}}{B})=\left\{\left(v_{i}, \mathscr{T}_{\mathscr{Q}\left(\check{d}_{k}\right)}\left(v_{i}\right), \mathscr{I}_{\mathscr{Q}\left(\check{d}_{k}\right)}\left(v_{i}\right)\right.\right.$, coefficient is given as $\delta_{\mathrm{NHSS}}^{1}((\wp, \stackrel{\dot{\mathrm{t}}}{A}),(\mathcal{Q}, \stackrel{\mathfrak{t}}{B}))$ and defined as follows:

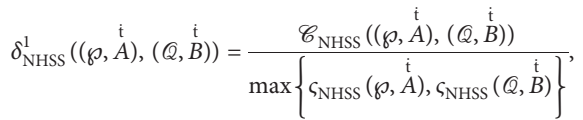

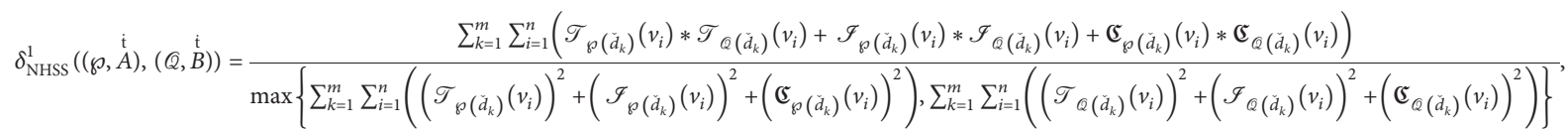

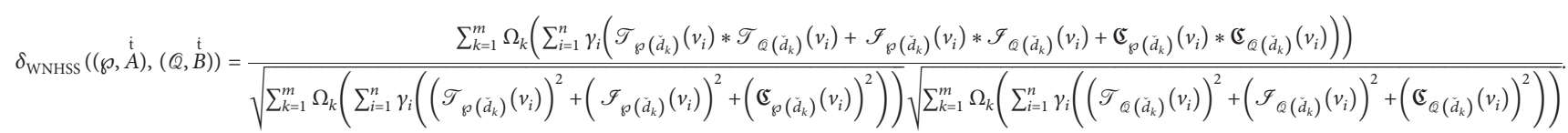


Theorem 2. Let $(\wp, \stackrel{\dot{t}}{A})=\left\{\left(v_{i}, \mathscr{T}_{\mathfrak{f}\left(\check{d}_{k}\right)}\left(v_{i}\right), \mathscr{J}_{\mathfrak{F}\left(\check{d}_{k}\right)}\left(v_{i}\right), \mathfrak{C}_{\mathfrak{\wp}\left(\check{d}_{k}\right)}\right.\right.$ $\left.\left.\left(v_{i}\right)\right) \quad \mid v_{i} \in \mathscr{U}\right\}$ and $(\mathscr{Q}, B)=\left\{\left(v_{i}, \mathscr{T}_{\mathscr{Q}\left(\check{d}_{k}\right)}\left(v_{i}\right), \mathscr{I}_{\mathscr{Q}\left(\check{d}_{k}\right)}\left(v_{i}\right)\right.\right.$, $\left.\left.\mathfrak{S}_{\mathscr{Q}\left(\breve{d}_{k}\right)}\left(v_{i}\right)\right) \mid v_{i} \in \mathscr{U}\right\}$ be two NHSSs. Then, CC between them satisfies the following properties:

(1) $0 \leq \delta_{N H S S}^{1}((\bullet, \stackrel{t}{A}),(\stackrel{i}{t}, \stackrel{t}{B})) \leq 1$

(2) $\delta_{\text {NHSS }}^{1}((\wp, A),(\mathscr{Q}, \stackrel{t}{B}))=\delta_{\text {NHSS }}^{1}((\mathbb{Q}, \stackrel{i}{B}),(\wp, \stackrel{t}{A}))$

(3) If $(\wp, A)=(\mathscr{Q}, B)$, that is, $\forall i, k, \mathscr{T}_{\wp\left(\check{d}_{k}\right)}\left(v_{i}\right)=$

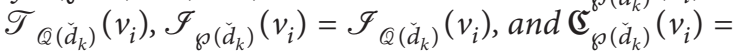
$\mathfrak{C}_{\mathscr{Q}\left(\check{d}_{k}\right)}\left(v_{i}\right)$, then $\delta_{\text {NHSS }}^{1}((\wp, \stackrel{t}{A}),(\mathscr{Q}, \stackrel{i}{B}))=1$

Proof. It is similar to Theorem 1.

It is very important to deliberate the weight of NHSS for practical fortitudes nowadays. Whenever experts regulate distinctive weights for every alternative, the choice might be dissimilar. So, it is, precisely, to plot the weights for experts preceding assembling a decision. Assume the weights of experts can be expressed as $\Omega=\left\{\Omega_{1}, \Omega_{2}, \Omega_{3}, \ldots, \Omega_{m}\right\}^{T}$, where $\Omega_{k}>0, \sum_{k=1}^{m} \Omega_{k}=1$. Similarly, assume the weights for subattributes as follows $\gamma=\left\{\gamma_{1}, \gamma_{2}, \gamma_{3}, \ldots, \gamma_{n}\right\}^{T}$, where $\gamma_{i}>0, \sum_{i=1}^{n} \gamma_{i}=1$.

Definition 12. Let $(\wp, \stackrel{\dot{\mathfrak{t}}}{A})=\left\{\left(v_{i}, \mathscr{T}_{\wp\left(\check{d}_{k}\right)}\left(v_{i}\right), \mathscr{I}_{\wp\left(\check{d}_{k}\right)}\left(v_{i}\right)\right.\right.$, $\left.\left.\mathfrak{C}_{\wp\left(\check{d}_{k}\right)}\left(v_{i}\right)\right) \mid v_{i} \in \mathscr{U}\right\} \quad$ and $\quad(\mathscr{Q}, \stackrel{\mathrm{t}}{B})=\left\{\left(v_{i}, \mathscr{T}_{\mathscr{Q}\left(\check{d}_{k}\right)}\left(v_{i}\right)\right.\right.$, $\left.\left.\mathscr{I}_{\mathscr{Q}\left(\check{d}_{k}\right)}\left(v_{i}\right), \mathfrak{C}_{\mathscr{Q}\left(\check{d}_{k}\right)}\left(v_{i}\right)\right) \mid v_{i} \in \mathscr{U}\right\}$ be two NHSSs. Then, WCC among them is expressed as $\delta_{\text {WNHSS }}((\wp, \stackrel{\mathfrak{t}}{A}),(\mathscr{Q}, \stackrel{\mathfrak{t}}{B}))$ and defined as follows:

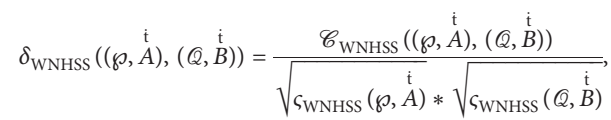

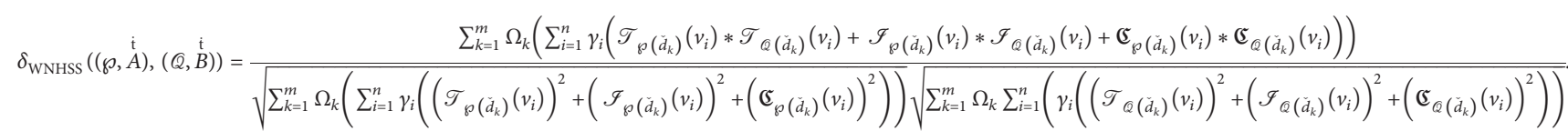

Definition 13. Let $(\wp, \stackrel{\mathfrak{t}}{A})=\left\{\left(v_{i}, \mathscr{T}_{\mathfrak{\wp}\left(\check{d}_{k}\right)}\left(v_{i}\right), \mathscr{I}_{\wp\left(\check{d}_{k}\right)}\left(v_{i}\right)\right.\right.$, $\left.\left.\mathfrak{C}_{\wp\left(\check{d}_{k}\right)}\left(v_{i}\right)\right) \mid v_{i} \in \mathscr{U}\right\}$ and $(\mathscr{Q}, B)=\left\{\left(v_{i}, \mathscr{T}_{\mathscr{Q}\left(\check{d}_{k}\right)}\left(v_{i}\right), \mathscr{J}_{\mathscr{Q}\left(\check{d}_{k}\right)}\right.\right.$ $\left.\left.\left(v_{i}\right), \mathfrak{夭}_{\mathscr{Q}\left(\check{d}_{k}\right)}\left(v_{i}\right)\right) \mid v_{i} \in \mathscr{U}\right\}$ be two NHSSs. Then, WCC among them is also given as $\delta_{\mathrm{WNHSS}}^{1}((\wp, \stackrel{\mathfrak{t}}{A}),(\mathcal{Q}, \stackrel{\mathfrak{t}}{B}))$ and defined as follows:

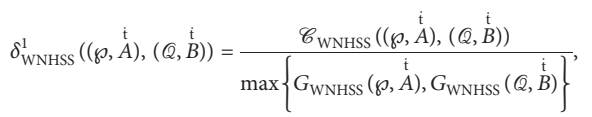

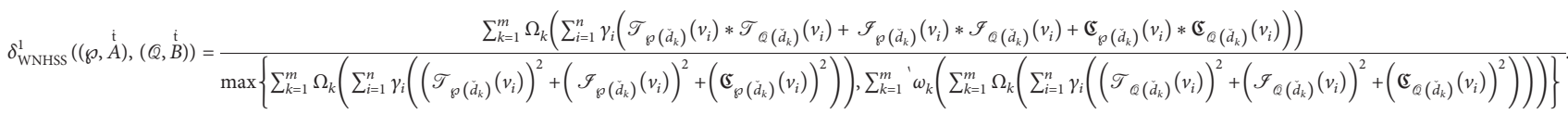

If we consider $\Omega=\left\{1 / m, 1 / m_{,} \ldots, 1 / m\right\}$ and $\gamma=\{1 / n$, $1 / n, \cdot \mathrm{t}, 1 / n\}$, then $\delta_{\text {WNHSS }}((\wp, A),(Q, B))_{\mathrm{t}}$ and $\delta_{\mathrm{WNHSS}}^{1}$ $((\wp, A),(\mathscr{Q}, B))$ are ${ }_{\mathrm{i}}$ reduced to $\delta_{\mathrm{WNHSS}}((\wp, A),(\mathscr{Q}, B))$ and $\delta_{\text {WNHSS }}^{1}((\wp, A),(Q, B))$, respectively.

Theorem 3. Let $\quad(\wp, \stackrel{i}{A})=\left\{\left(v_{i}, \mathscr{T}_{\mathscr{\wp}\left(\breve{q}_{k}\right)}\left(v_{i}\right), \quad \mathscr{I}_{\mathfrak{Q}\left(\check{d}_{k}\right)}\left(v_{i}\right)\right.\right.$, $\left.\left.\mathfrak{夭}_{\wp\left(\check{d}_{k}\right)}\left(v_{i}\right)\right) \mid v_{i} \in \mathscr{U}\right\} \quad$ and $\quad(\mathscr{Q}, B)=\left\{\left(v_{i}, \mathscr{T}_{\mathscr{Q}\left(\check{d}_{k}\right)}\left(v_{i}\right)\right.\right.$, $\left.\left.\mathscr{I}_{\mathbb{Q}\left(\breve{d}_{k}\right)}\left(v_{i}\right), \mathfrak{C}_{\mathscr{Q}\left(\check{d}_{k}\right)}\left(v_{i}\right)\right) \mid v_{i} \in \mathscr{U}\right\}$ be two NHSSs; then, WCC between them satisfies the following properties:
(1) $0 \leq \delta_{\text {WNHSS }}((\wp, \stackrel{\dot{t}}{A}),(\mathscr{Q}, \stackrel{\dot{t}}{B})) \leq 1$

(2) $\delta_{\text {WNHSS }}((\wp, \stackrel{i}{A}),(\stackrel{i}{t}, \stackrel{i}{B}))=\delta_{\text {WNHSS }}((\stackrel{i}{Q}, \stackrel{i}{B}),(\stackrel{i}{\stackrel{t}{A}}))$

(3) If $(\wp, A)=(\mathscr{Q}, B)$, that is, $\forall i, k, \mathscr{T}_{\wp\left(\check{d}_{k}\right)}\left(v_{i}\right)=$ $\mathscr{T}_{\mathscr{Q}\left(\breve{d}_{k}\right)}\left(v_{i}\right), \mathscr{I}_{\mathfrak{f}\left(\check{d}_{k}\right)}\left(v_{i}\right)=\mathscr{I}_{\mathscr{Q}\left(\breve{d}_{k}\right)}\left(v_{i}\right)$, and $\mathfrak{C}_{\mathfrak{f}\left(\dot{d}_{k}\right)}\left(v_{i}\right)=$ $\mathfrak{C}_{\mathbb{Q}\left(\check{d}_{k}\right)}\left(v_{i}\right)$, then $\delta_{\text {WNHSS }}((\wp, \stackrel{\dot{t}}{A}),(\mathscr{Q}, \stackrel{\dot{t}}{B}))=1$

Proof. $\quad \delta_{\text {WNHSS }}((\wp, \stackrel{\mathfrak{t}}{A}),(\mathcal{Q}, \stackrel{\mathfrak{t}}{B})) \geq 0$ is trivial $_{\mathfrak{i}}$ and here, we only need to prove that $\delta_{\mathrm{WNHSS}}((\wp, A),(Q, B)) \leq 1$.

We have 
$\mathscr{C}_{\text {wNHSS }}((\wp, \ddot{A}),(\mathscr{Q}, \ddot{B}))$

$$
\begin{aligned}
& =\sum_{k=1}^{m} \Omega_{k}\left(\sum_{i=1}^{n} \gamma_{i}\left(\mathscr{T}_{\mathscr{P}\left(\check{d}_{k}\right)}\left(v_{i}\right) * \mathscr{T}_{\mathscr{Q}\left(\check{d}_{k}\right)}\left(v_{i}\right)+\mathscr{I}_{\mathfrak{\wp}\left(\check{d}_{k}\right)}\left(v_{i}\right) * \mathscr{I}_{\mathbb{Q}\left(\check{d}_{k}\right)}\left(v_{i}\right)+\mathfrak{C}_{\mathfrak{f}\left(\check{d}_{k}\right)}\left(v_{i}\right) * \widetilde{C}_{\mathscr{Q}\left(\check{d}_{k}\right)}\left(v_{i}\right)\right)\right) \\
& =\sum_{k=1}^{m} \Omega_{k}\left(\gamma_{1}\left(\mathscr{T}_{\mathscr{V}\left(\check{d}_{k}\right)}\left(v_{1}\right) * \mathscr{T}_{\mathscr{Q}\left(\check{d}_{k}\right)}\left(v_{1}\right)+\mathscr{I}_{\mathfrak{f}\left(\check{d}_{k}\right)}\left(v_{1}\right) * \mathscr{I}_{\mathscr{Q}\left(\check{d}_{k}\right)}\left(v_{1}\right)+\mathfrak{C}_{\mathfrak{Q}\left(\check{d}_{k}\right)}\left(v_{1}\right) * \mathfrak{C}_{\mathscr{Q}\left(\check{d}_{k}\right)}\left(v_{1}\right)\right)\right) \\
& +\sum_{k=1}^{m} \Omega_{k}\left(\gamma_{2}\left(\mathscr{T}_{\mathfrak{\wp}\left(\check{d}_{k}\right)}\left(v_{2}\right) * \mathscr{T}_{\mathscr{Q}\left(\check{d}_{k}\right)}\left(v_{2}\right)+\mathscr{I}_{\mathfrak{\wp}\left(\check{d}_{k}\right)}\left(v_{2}\right) * \mathscr{I}_{\mathscr{Q}\left(\check{d}_{k}\right)}\left(v_{2}\right)+\mathfrak{C}_{\mathfrak{\wp}\left(\check{d}_{k}\right)}\left(v_{2}\right) * \mathbb{C}_{\mathscr{Q}\left(\check{d}_{k}\right)}\left(v_{2}\right)\right)\right) \\
& + \\
& \vdots \\
& + \\
& \sum_{k=1}^{m} \Omega_{k}\left(\gamma_{n}\left(\mathscr{T}_{\mathfrak{\wp}\left(\check{d}_{k}\right)}\left(v_{n}\right) * \mathscr{T}_{\mathscr{Q}\left(\check{d}_{k}\right)}\left(v_{n}\right)+\mathscr{I}_{\mathfrak{\wp}\left(\check{d}_{k}\right)}\left(v_{n}\right) * \mathscr{I}_{\mathscr{Q}\left(\check{d}_{k}\right)}\left(v_{n}\right)+\mathfrak{C}_{\mathscr{\wp}\left(\check{d}_{k}\right)}\left(v_{n}\right) * \mathfrak{C}_{\mathscr{Q}\left(\check{d}_{k}\right)}\left(v_{n}\right)\right)\right)
\end{aligned}
$$

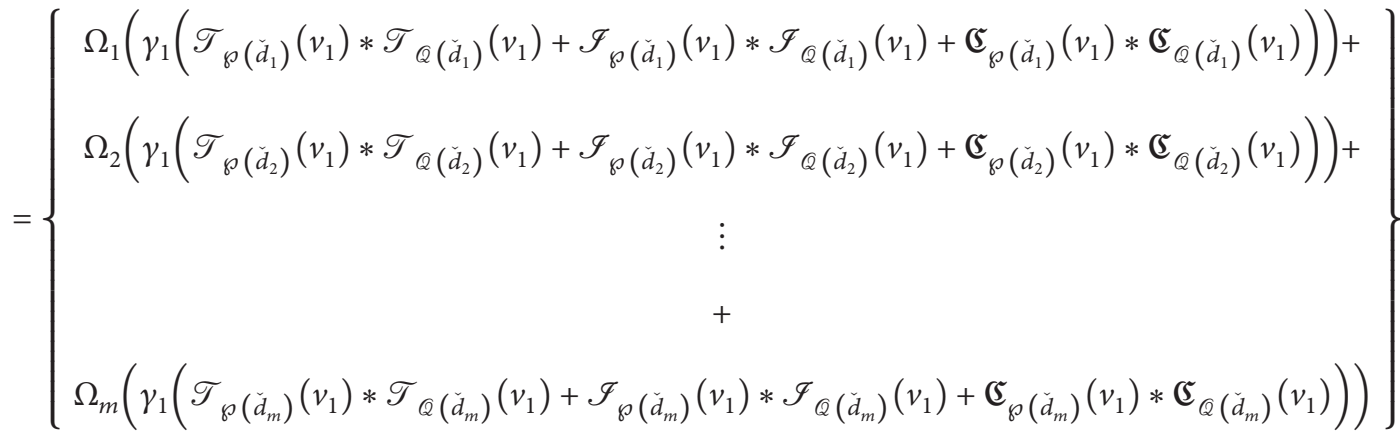

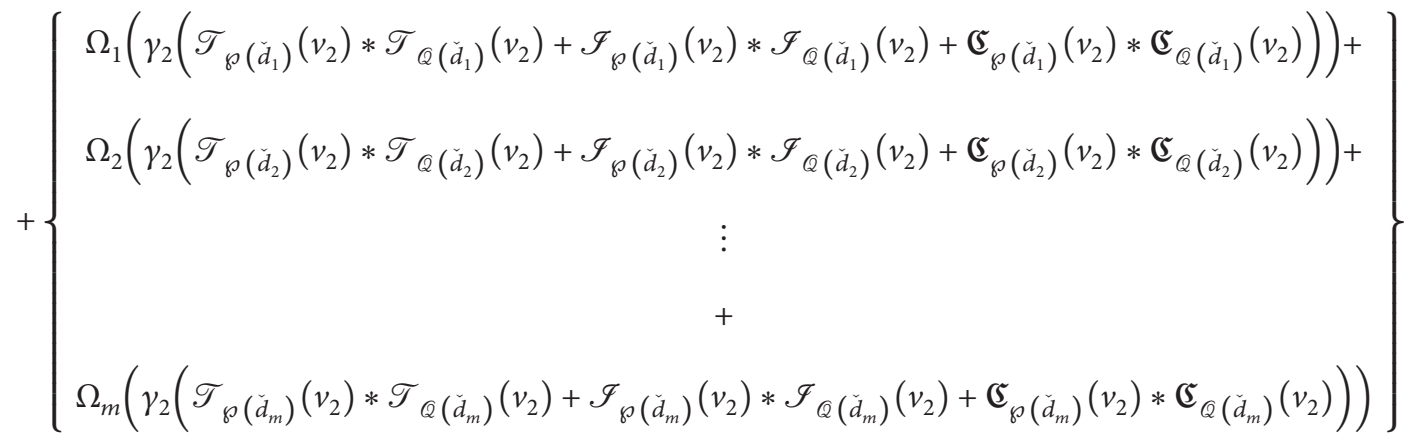




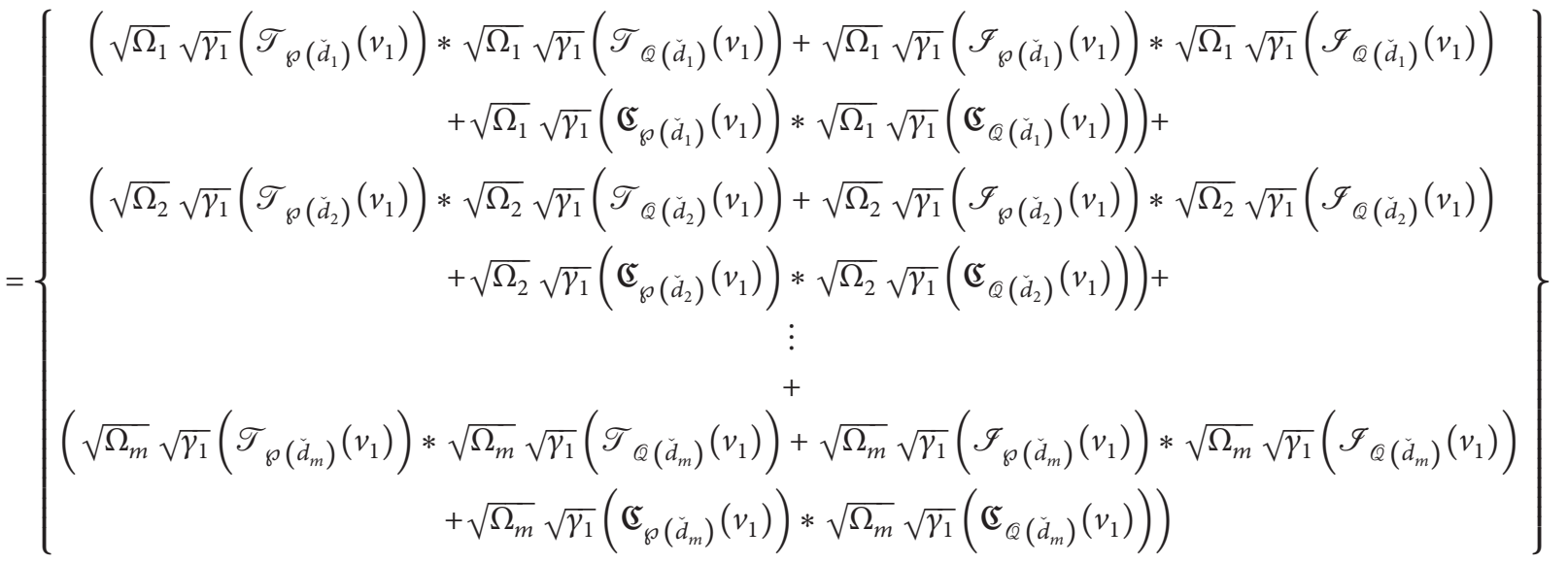

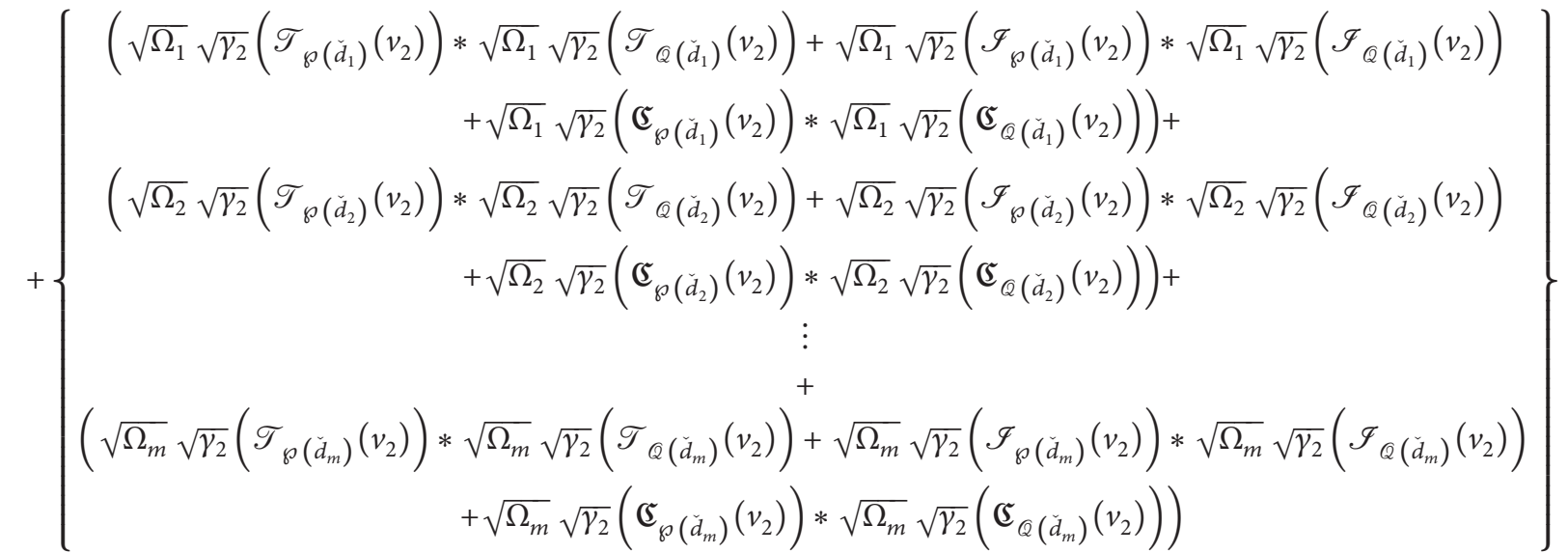

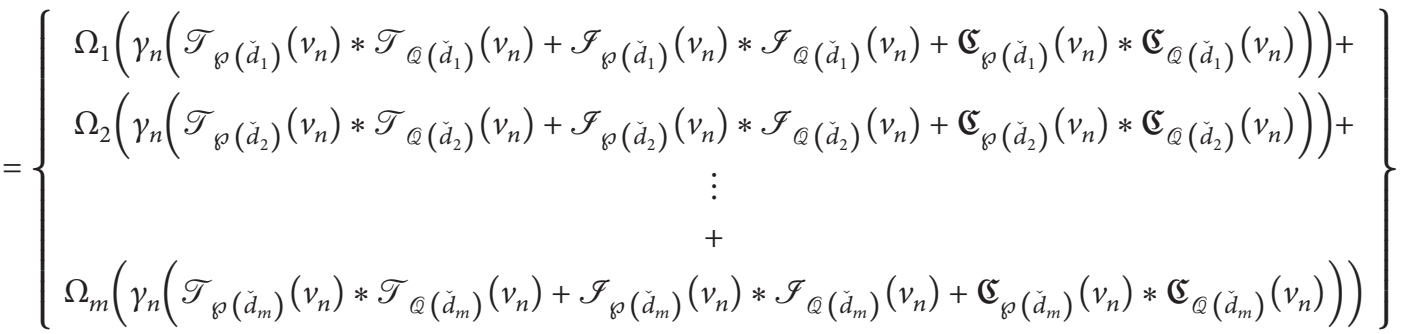

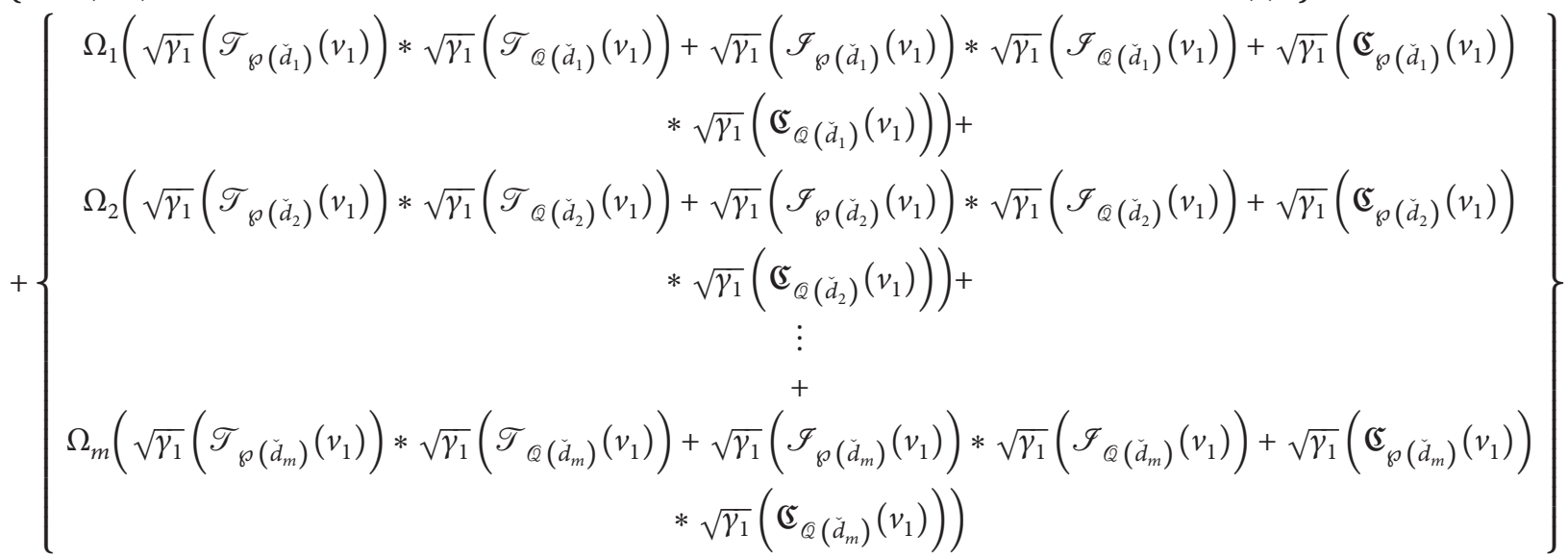




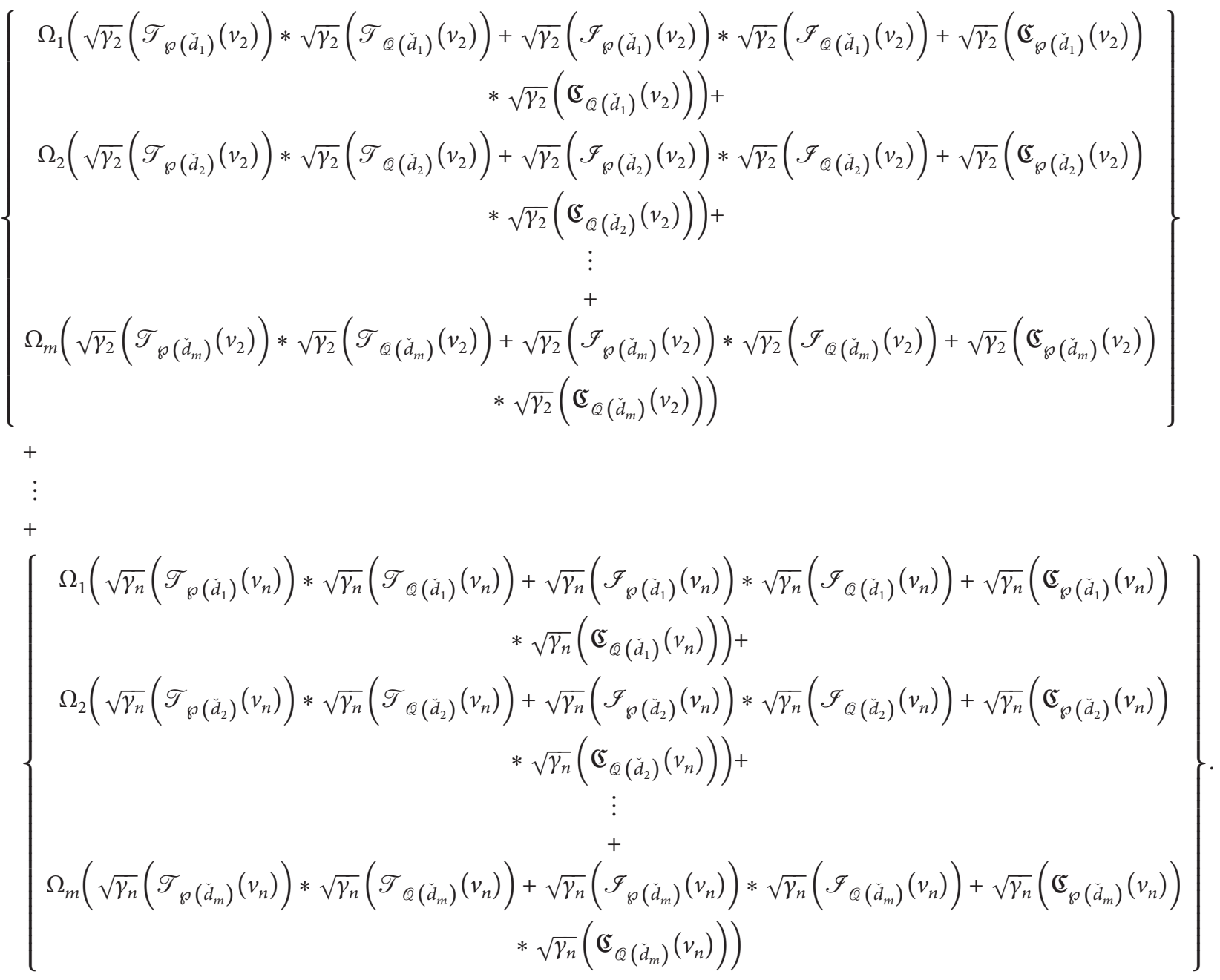

By using Cauchy Schwarz inequality, we obtain

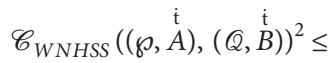

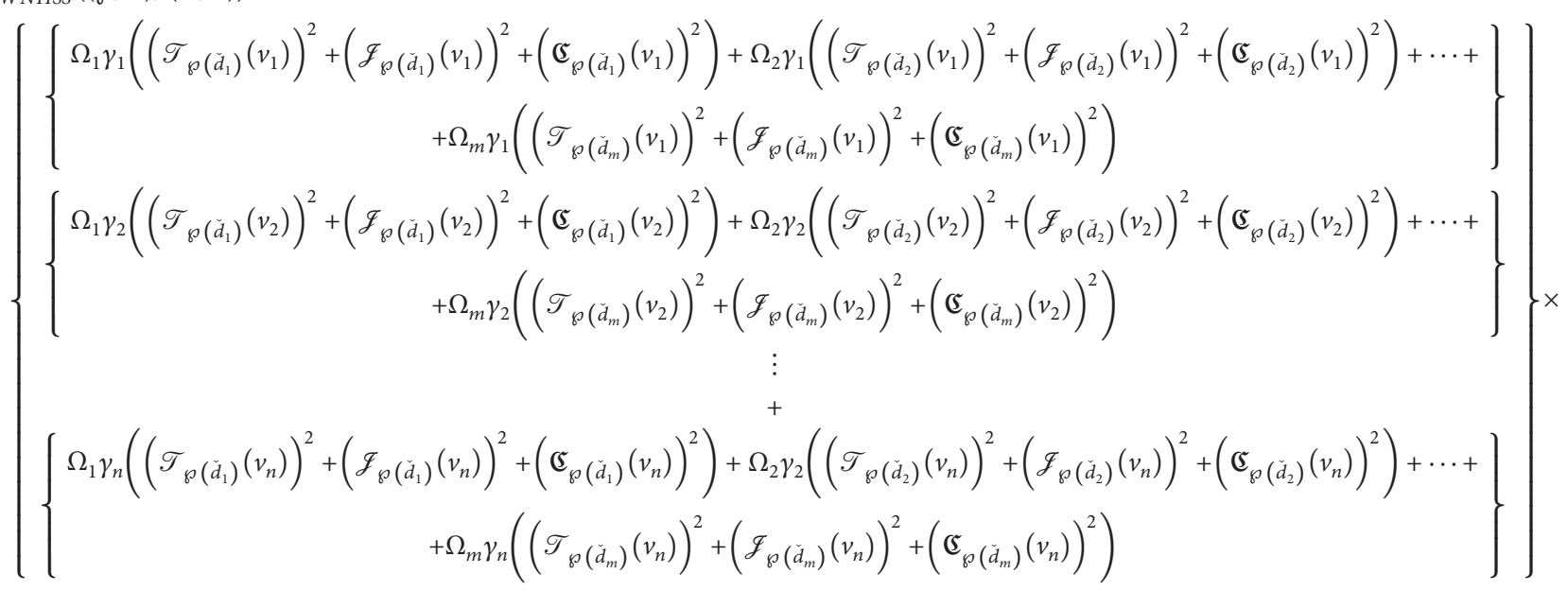




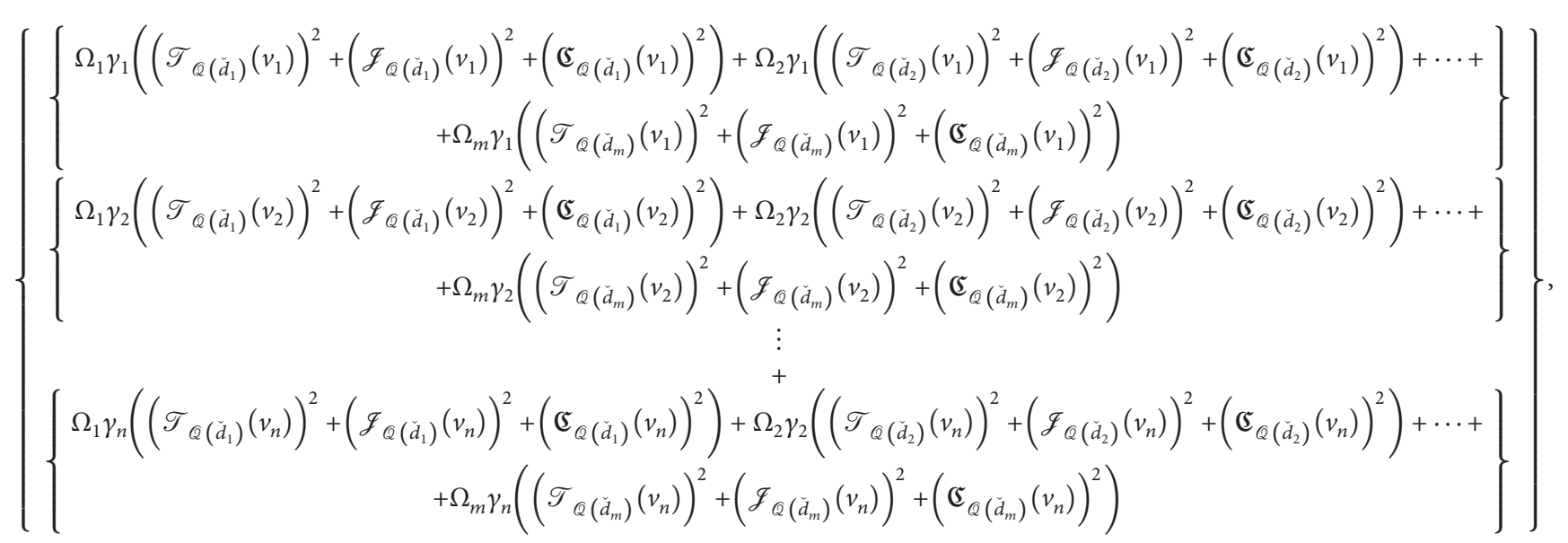

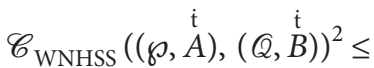

$$
\begin{aligned}
& \sum_{k=1}^{m} \Omega_{k}\left(\sum_{i=1}^{n} \gamma_{i}\left(\left(\mathscr{T}_{\wp\left(\check{d}_{k}\right)}\left(v_{i}\right)\right)^{2}+\left(\mathscr{I}_{\wp\left(\check{d}_{k}\right)}\left(v_{i}\right)\right)^{2}+\left(\mathfrak{夭}_{\wp\left(\check{d}_{k}\right)}\left(v_{i}\right)\right)^{2}\right)\right) \\
& \times \sum_{k=1}^{m} \Omega_{k}\left(\sum_{i=1}^{n} \gamma_{i}\left(\left(\mathscr{T}_{\mathscr{Q}\left(\check{d}_{k}\right)}\left(v_{i}\right)\right)^{2}+\left(\mathscr{I}_{\mathscr{Q}\left(\check{d}_{k}\right)}\left(v_{i}\right)\right)^{2}+\left(\mathfrak{S}_{\mathscr{Q}\left(\check{d}_{k}\right)}\left(v_{i}\right)\right)^{2}\right)\right)
\end{aligned}
$$

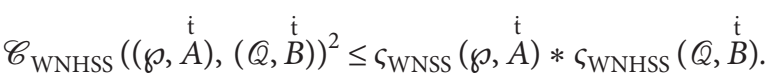

Therefore, $\mathscr{C}_{\text {WNHSS }}((\wp, \stackrel{\dot{t}}{\mathrm{~A}}),(\stackrel{\dot{\mathrm{t}}}{\mathrm{B}})) \leq$ Proof. The proof is straightforward.

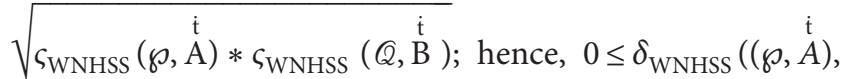
$(Q, \stackrel{\dot{\mathfrak{t}}}{\mathrm{Q}})) \leq 1$.

$\delta_{\text {WNHSS }}((\wp, \stackrel{\stackrel{\mathrm{t}}{A}}{)}),(\mathcal{Q}, \stackrel{\mathrm{t}}{B}))$

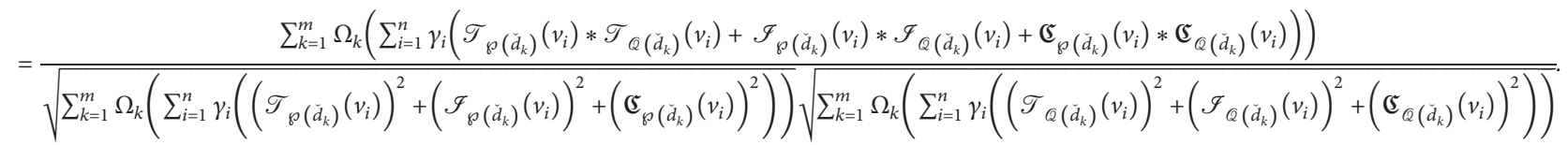

As we know that $\mathscr{T}_{\wp\left(\check{d}_{k}\right)}\left(v_{i}\right)=\mathscr{T}_{\mathscr{Q}\left(\check{d}_{k}\right)}\left(v_{i}\right), \mathscr{I}_{\wp\left(\check{d}_{k}\right)}\left(v_{i}\right)=$

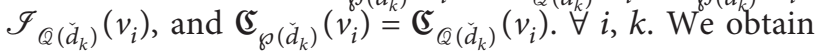

$\delta_{\text {WNHSS }}((\wp, \stackrel{\mathrm{t}}{A}),(\mathscr{Q}, \stackrel{\mathrm{t}}{B}))$

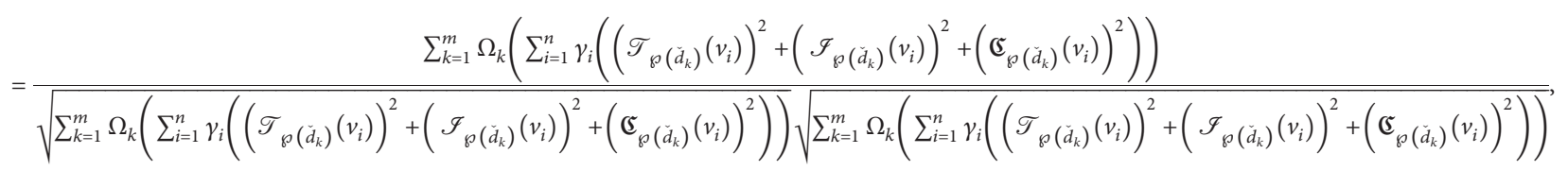

$\delta_{\text {WNHSS }}((\wp, \stackrel{\mathfrak{t}}{A}),(\mathcal{Q}, \stackrel{\stackrel{t}{ }}{)}))=1$. 
Definition 14. Let $\widetilde{J}_{\check{d}_{k}}=\left\langle\mathscr{T}_{\mathscr{F}\left(\check{d}_{i j}\right)}, \mathscr{J}_{\mathscr{F}\left(\check{d}_{i j}\right)}\right\rangle, \mathfrak{E}_{\mathscr{F}\left(\check{d}_{i j}\right)}, \mathfrak{J}_{\check{d}_{11}}=$ $\left\langle\mathscr{T}_{\mathscr{F}\left(\check{d}_{11}\right)}, \quad \mathscr{J}_{\mathscr{F}\left(\check{d}_{11}\right)}, \mathfrak{S}_{\mathscr{F}\left(\check{d}_{11}\right)}\right\rangle$, and $\widetilde{\mathfrak{J}}_{\check{d}_{12}}=\left\langle\mathscr{T}_{\mathscr{F}\left(\check{d}_{12}\right)}, \mathscr{J}_{\mathscr{F}\left(\check{d}_{12}\right)}\right.$, $\left.\mathfrak{S}_{\mathscr{F}\left(\check{d}_{12}\right)}\right\rangle$ be three NHSNs and $\alpha$ be a positive real number; by algebraic norms, we have

(1) $\mathfrak{J}_{\check{d}_{11}} \oplus \widetilde{\mathfrak{J}}_{\check{d}_{12}}=\left\langle\mathscr{T}_{\mathscr{F}\left(\check{d}_{11}\right)}+\mathscr{T}_{\mathscr{F}\left(\check{d}_{12}\right)}-\mathscr{T}_{\mathscr{F}\left(\check{d}_{11}\right)} \mathscr{T}_{\mathscr{F}\left(\check{d}_{12}\right)}\right.$, $\mathscr{J}_{\mathscr{F}\left(\check{d}_{11}\right)} \mathscr{J}_{\mathscr{F}\left(\check{d}_{12}\right)}, \boldsymbol{⿰}_{\mathscr{F}\left(\check{d}_{11}\right)} \boldsymbol{\mathfrak { S }}_{\mathscr{F}\left(\check{d}_{12}\right)}>$

(2) $\widetilde{J}_{\check{d}_{11}} \otimes \widetilde{\mathfrak{J}}_{\check{d}_{12}}=\left\langle\mathscr{T}_{\mathscr{F}\left(\check{d}_{11}\right)} \mathscr{T}_{\mathscr{F}\left(\check{d}_{12}\right)}, \mathscr{J}_{\mathscr{F}\left(\check{d}_{11}\right)}+\mathscr{J}_{\mathscr{F}\left(\check{d}_{12}\right)}-\right.$ $\mathscr{J}_{\mathscr{F}\left(\check{d}_{11}\right)} \mathscr{J}_{\mathscr{F}\left(\check{d}_{12}\right)}, \mathfrak{S}_{\mathscr{F}\left(\check{d}_{11}\right)}+\mathfrak{⿰}_{\mathscr{F}\left(\check{d}_{12}\right)}-\mathfrak{G}_{\mathscr{F}\left(\check{d}_{11}\right)} \boldsymbol{夭}_{\mathscr{F}\left(\check{d}_{12}\right)}>$

(3) $\alpha \mathfrak{\Im}_{\check{d}_{k}}=<1-\left(1-\mathscr{T}_{\check{d}_{k}}\right)^{\alpha}, \mathscr{J}_{\check{d}_{k}}{ }^{\alpha}, \mathfrak{C}_{\check{d}_{k}}{ }^{\alpha}>$
(4) $\mathfrak{\Im}_{\check{d}_{k}}^{\alpha}=<\mathscr{T}_{\check{d}_{k}}^{\alpha}, 1-\left(1-\mathscr{J}_{\check{d}_{k}}\right)^{\alpha}, 1-\left(1-\mathfrak{C}_{\check{d}_{k}}\right)^{\alpha}>$

We develop some AOs based on the above-defined operational laws for the collection of NHSNs $\Delta$.

Definition 15. Let $\widetilde{\mathfrak{J}}_{\check{d}_{k}}=\left\langle\mathscr{T}_{\mathscr{F}\left(\check{d}_{i j}\right)}, \mathscr{J}_{\mathscr{F}\left(\check{d}_{i j}\right)}, \mathfrak{夭}_{\mathscr{F}\left(\check{d}_{i j}\right)}\right\rangle$ be an NHSN, and $\Omega_{i}$ and $\gamma_{j}$ are weight vector for expert's and subattributes of the considered attributes correspondingly along with specified circumstances $\Omega_{i}>0, \sum_{i=1}^{n} \Omega_{i}=1$, $\gamma_{j}>0, \sum_{j=1}^{m} \gamma_{j}=1$. Then, NHSWA operator is defined as follows.

NHSWA: $\Delta^{n} \longrightarrow \Delta$ is defined as follows:

$$
\begin{aligned}
\operatorname{NHSWA}\left(\mathfrak{\Im}_{\check{d}_{11}}, \mathfrak{\Im}_{\check{d}_{12}}, \ldots, \mathfrak{\Im}_{\check{d}_{n m}}\right) & =\oplus_{j=1}^{m} \gamma_{j}\left(\oplus_{i=1}^{n} \Omega_{i} \mathfrak{\Im}_{\check{d}_{i j}}\right) \\
& =\left\langle 1-\prod_{j=1}^{m}\left(\prod_{i=1}^{n}\left(1-\mathscr{T}_{\check{d}_{i j}}\right)^{\Omega_{i}}\right)^{\gamma_{j}}, \prod_{j=1}^{m}\left(\prod_{i=1}^{n}\left(\mathscr{J}_{\check{d}_{i j}}\right)^{\Omega_{i}}\right)^{\gamma_{j}} \prod_{j=1}^{m}\left(\prod_{i=1}^{n}\left(\mathfrak{E}_{\check{d}_{i j}}\right)^{\Omega_{i}}\right)^{\gamma_{j}}\right\rangle .
\end{aligned}
$$

Definition 16. Let $\widetilde{J}_{\check{d}_{k}}=\left\langle\mathscr{T}_{\mathscr{F}\left(\check{d}_{i j}\right)}, \mathscr{J}_{\mathscr{F}\left(\check{d}_{i j}\right)}, \mathfrak{S}_{\mathscr{F}\left(\check{d}_{i j}\right)}\right\rangle$ be an NHSN, and $\Omega_{i}$ and $\gamma_{j}$ are weight vector for expert's and subattributes of the considered attributes correspondingly along with specified circumstances $\Omega_{i}>0, \sum_{i=1}^{n} \Omega_{i}=1$, and $\gamma_{j}>0, \sum_{j=1}^{m} \gamma_{j}=1$. Then, NHSWG operator is defined as follows.

NHSWG: $\Delta^{n} \longrightarrow \Delta$ defined as follows:

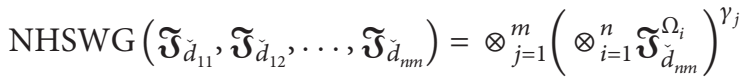

$$
\begin{aligned}
& \operatorname{NHSWG}\left(\mathfrak{J}_{\check{d}_{11}}, \mathfrak{J}_{\check{d}_{12}}, \ldots, \mathfrak{J}_{\check{d}_{n m}}\right)=\left\langle\prod_{j=1}^{m}\left(\prod_{i=1}^{n}\left(\mathscr{T}_{\check{d}_{i j}}\right)^{\Omega_{i}}\right)^{\gamma_{j}}, 1-\prod_{j=1}^{m}\left(\prod_{i=1}^{n}\left(1-\mathscr{J}_{\check{d}_{i j}}\right)^{\Omega_{i}}\right)^{\gamma_{j}}, 1-\prod_{j=1}^{m}\left(\prod_{i=1}^{n}\left(1-\mathfrak{S}_{\check{d}_{i j}}\right)^{\Omega_{i}}\right)^{\gamma_{j}}\right\rangle .
\end{aligned}
$$

\section{TOPSIS Approach on NHSS for MCGDM Problem Based on the Correlation Coefficient}

In this section, we are going to develop a methodology for solving DM issues through continuing the TOPSIS technique for NHSS information according to CC. Hwang and Yoon [51] progressed the TOPSIS methodology and utilized it to promote the order of evaluation components of the PIA and NIA for DM complications. Through the use of the presented approach, we will find the best possible choices which have the smallest and largest distances to PIS and NIS. The TOPSIS method ensures that the correlation measure can be utilized to differentiate PIA and NIA by choosing positions. Generally, investigators are using the TOPSIS approach to find CC along with distinctive distance forms. The TOPSIS method along with CC is superior for locating closeness coefficients instead of distance as well as similarity measures. Since the correlation measure holds the direct association among the aspects restrained, by utilizing the established CC, a TOPSIS method is presented to choose the most appropriate choice.
4.1. Proposed Decision-Making Approach. Let $\mathscr{X}=\left\{\mathscr{X}_{1}, \mathscr{X}_{2}\right.$, $\left.\mathscr{X}_{3}, \ldots, X_{s}\right\}$ be a collection of " $s$ " alternatives for assessment under the group of experts $Q=\left\{Q^{1}, Q^{2}, Q^{3}, \ldots, Q^{n}\right\}$ with weights $\Omega=\left(\Omega_{1}, \Omega_{1}, \ldots, \Omega_{n}\right)^{T}$ and $\Omega_{i}>0, \quad \sum_{i=1}^{n} \Omega_{i}=1$. Consider $\mathbb{Q}=\left\{d_{1}, d_{2}, \ldots, d_{m}\right\}$ be a set of attributes with their corresponding subattributes such as $\mathbf{Q}^{\prime}=\left\{\left(d_{1 \rho} \times d_{2 \rho} \times\right.\right.$ $\left.\cdots \times d_{m \rho}\right)$ for all $\left.\rho \in\{1,2, \ldots, t\}\right\}$ with weights $\gamma=\left(\gamma_{1 \rho}, \gamma_{2 \rho}\right.$, $\left.\gamma_{3 \rho}, \ldots, \gamma_{m \rho}\right)^{T}$ such as $\gamma_{\rho}>0, \sum_{\rho=1}^{t} \gamma_{\rho}=1$, and can be stated as $\mathfrak{\Omega}^{\prime}=\left\{\check{d}_{\partial}: \partial \in\{1,2, \ldots, k\}\right\}$. The group of experts $\left\{\mathscr{Q}^{i}: i=1\right.$, $2, \ldots, n\}$ evaluate the alternatives $\left\{\mathscr{X}_{(z)}: z=1,2, \ldots, s\right\}$ under the desired subattributes of the considered parameters $\left\{\check{d}_{\partial}: \partial=1,2, \ldots, k\right\}$ given in the form of NHSNs such as $\left(\aleph_{\tilde{d}_{i k}}^{(z)}\right)_{n \times \partial}=\left(\mathscr{T}_{\tilde{d}_{i k}}^{(z)}, \mathscr{I}_{\mathfrak{d}_{i k}}^{(z)}, \mathfrak{C}_{\breve{d}_{i k}}^{(z)}\right)_{n \times \partial}, \quad$ where $0 \leq \mathscr{T}_{\check{d}_{i k}}^{(z)}, \mathscr{I}_{\check{d}_{i k}}^{(z)}, \mathfrak{S}_{\breve{d}_{i k}}^{(z)} \leq 1$ and $0 \leq \mathscr{T}_{\check{d}_{i k}}^{(z)}+\mathscr{I}_{\breve{d}_{i k}}^{(z)}+\mathfrak{C}_{\breve{d}_{i k}}^{(z)} \leq 3$ for all $i, k$.

Step 1: development of decision matrices for each alternative $\left\{\mathscr{X}_{(z)}: z=1,2, \ldots, s\right\}$ by using subattributes of 
the given attributes in the form of NHSNs such as follows:

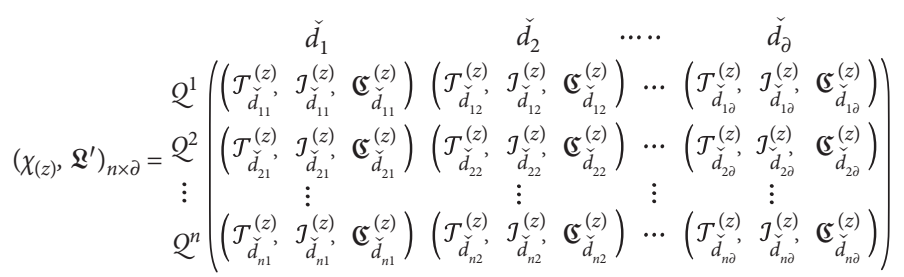

Step 2: normalize the aggregated decision matrices by transforming cost type subattributes to benefit type subattribute using the normalization rule:

$$
\mathscr{X}_{i j}= \begin{cases}\mathfrak{J}_{\check{d}_{i j}}^{c}, & \text { cost type parameter, } \\ \mathfrak{J}_{\check{d}_{i j}}, & \text { benefit type parameter, }\end{cases}
$$

where $\mathfrak{J}_{\breve{d}_{i j}}^{\mathcal{c}}=\left\langle 1-\mathfrak{S}_{\check{d}_{i j}}, \mathscr{J}_{\check{d}_{i j}}, \mathscr{T}_{\breve{d}_{i j}}\right\rangle$, which represents the complement of $\left\langle\mathscr{T}_{\dot{d}_{i j}}, \mathscr{J}_{\dot{d}_{i j}}, \mathfrak{S}_{\mathfrak{d}_{i j}}\right\rangle$.

Step 3: development of weighted decision matrix for each alternative $\overline{\mathscr{X}}_{z}=\left(\overline{\mathfrak{J}}_{d_{i j}}^{(z)}\right)_{n \times \partial}$, where

$$
\begin{aligned}
\overline{\mathfrak{J}}_{\dot{d}_{i j}}^{(z)}= & \gamma_{j} \Omega_{i} \widetilde{\mathfrak{J}}_{\check{d}_{i j}}^{(z)} \\
& \cdot\left\langle 1-\prod_{j=1}^{m}\left(\prod_{i=1}^{n}\left(1-\mathscr{T}_{\check{d}_{i j}}\right)^{\Omega_{i}}\right)^{\gamma_{j}}, \prod_{j=1}^{m} \prod_{i=1}^{n}\left(\mathscr{J}_{\check{d}_{i j}}\right)^{\Omega_{i} \gamma_{j}}, \prod_{j=1}^{m}\left(\prod_{i=1}^{n}\left(\mathfrak{S}_{\check{d}_{i j}}\right)^{\Omega_{i}}\right)^{\gamma_{j}}\right\rangle \\
= & \left(\overline{\mathscr{T}}_{\check{d}_{i j}}^{(z)}, \overline{\mathscr{J}}_{\check{d}_{i j}}^{(z)}, \overline{\mathfrak{C}}_{\check{d}_{i j}}^{(z)}\right) .
\end{aligned}
$$

Here, $\Omega_{i}$ and $\gamma_{j}$ expressed the weights of experts and subattributes.

Step 4: to determine the PIA and NIA compute indices by utilizing equation (12), such as $\operatorname{argmax}_{z}\left\{\theta_{i j}^{(z)}\right\}$ and $g_{i j}=\arg \min _{z}\left\{\theta_{i j}^{(z)}\right\}$,

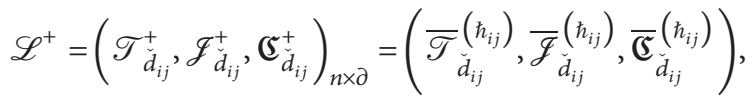

$$
\mathscr{L}^{-}=\left(\mathscr{T}_{\check{d}_{i j}}^{-}, \mathscr{J}_{\check{d}_{i j}}^{-}, \mathfrak{S}_{\breve{d}_{i j}}^{-}\right)_{n \times \partial}=\left(\overline{\mathscr{T}}_{\check{d}_{i j}}^{\left(g_{i j}\right)}, \overline{\mathscr{J}}_{\breve{d}_{i j}}^{\left(g_{i j}\right)}, \overline{\mathfrak{C}}_{\breve{d}_{i j}}\left(g_{i j}\right)\right) .
$$

Step 5: consider $\overline{\mathscr{X}}_{z}$ and PIA $\mathscr{L}^{+}$analyze the CC such as follows:

$$
\begin{aligned}
& p^{(z)}=\delta_{\mathrm{NHSS}}\left(\overline{\mathscr{X}}_{z}, \mathscr{L}^{+}\right)=\frac{\mathscr{C}_{\mathrm{NHSS}}\left(\overline{\mathscr{X}}_{z}, \mathscr{L}^{+}\right)}{\sqrt{\varsigma_{\mathrm{NHSS}} \mathscr{X}_{z} * \varsigma_{\mathrm{NHSS}} \mathscr{L}^{+}}}
\end{aligned}
$$

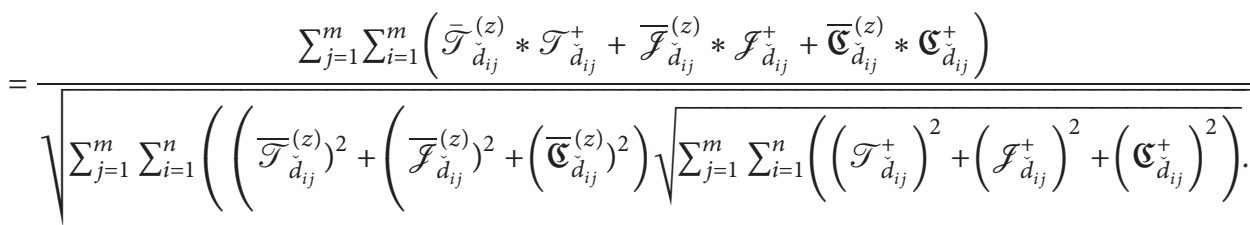


Step 6: consider $\bar{X}_{z}$ and NIA $\mathscr{L}^{-}$analyze the CC such as follows:

$$
\begin{aligned}
& q^{(z)}=\delta_{\mathrm{NHSS}}\left(\overline{\mathscr{X}}_{z}, \mathscr{L}^{-}\right)=\frac{\mathscr{C}_{\mathrm{NHSS}}\left(\overline{\mathscr{X}}_{z}, \mathscr{L}^{-}\right)}{\sqrt{\varsigma_{\mathrm{NHSS}} \mathscr{X}_{z} * \varsigma_{\mathrm{NHSS}} \mathscr{L}^{-}}}
\end{aligned}
$$

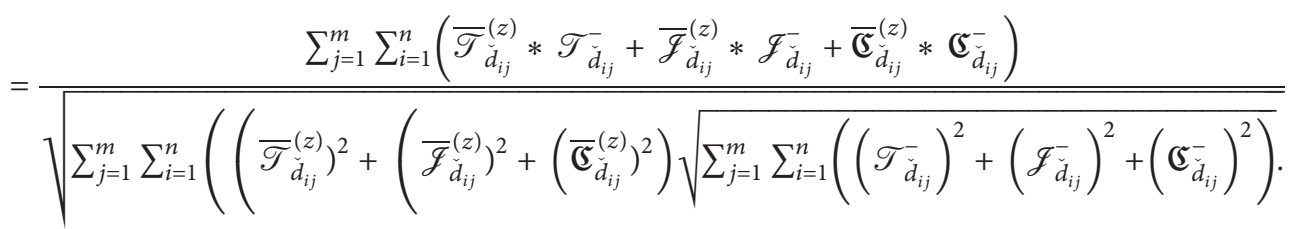

Step 7: for the alternatives, ranking computes the closeness coefficient:

$$
\mathscr{R}^{(z)}=\frac{\mathscr{K}\left(\overline{\mathscr{X}}_{z}, \mathscr{L}^{-}\right)}{\mathscr{K}\left(\overline{\mathscr{X}}_{z}, \mathscr{L}^{+}\right)+\mathscr{K}\left(\overline{\mathscr{X}}_{z}, \mathscr{L}^{-}\right)} .
$$

where

$$
\mathscr{K}\left(\overline{\mathscr{X}}_{z}, \mathscr{L}^{-}\right)=1-q^{(z)} \text { and } \mathscr{K}\left(\overline{\mathscr{X}}_{z}, \mathscr{L}^{+}\right)=1-p^{(z)} .
$$

Step 8: pick the alternate with the largest closeness coefficient.

Step 9: rank the alternatives.

Graphical representation of the above presented TOPSIS algorithm is given in Figure 1.

\subsection{Application of the Proposed MCGDM Technique for the Selection of Hand Sanitizer}

4.2.1. Case Study. Sanitization is the most important process because direct contact along with airborne microorganism droplets caused by sneezing and sneezing can without problems be infected. Particularly, when a pandemic breaks out, it is necessary to interrupt the overall chain of transmitting of the virus by proper hand sterilization trials. This will be accomplished by employing the right to use isolation as well as accommodating emergency care possessions, such as handling sterilizing in psychiatric divisions as well as public places. The assumption of sanitizers simply relies on the use of multipurpose sanitizers developed in various types and varieties, nominative antibacterial soaps and waterbased or alcohol-based sanitizers, which have been common in hospitals. Until now, the most helpful hand sanitizer product is the alcohol-based formulation, which could include $62 \%$ to $95 \%$ alcohol, since it can convert microbic proteins and have the ability to inactivate infections. The reassessment of this system of rules is expounded to the information in PubMed. It is going to examine the variety of available sanitizers along with their efficiency plus formulation features, contrary effects, and approvals to improve preparation proficiency along with safety. Furthermore, this article concentrates on the efficiency of alcohol-based sanitizers against disease. Up to now, the most effective hand sanitizer overall has been formulated based on alcoholic brews, which conquer $62 \%$ to $95 \%$ of alcoholic beverages because it can formalize microbial proteins and $[52,53]$ can be disabled. Due to its intoxicated alcohol content, the current expression gives some rival as well as concerns about safety disadvantages along with skin cytotoxicity [54]. Current research objectives scrutinize a variety of accessible sanitizers, as well as their effectiveness against the redeeming coronavirus, besides strategies and findings as well as ideas for reinforcing the formation of saviors from accessible hands.

Hand sanitizers may be a liquid or gel, mainly used to cut down transmittable diseases on the hands. In most organizations, alcohol-based sanitizers are superior for cleaning. The overall concentrates for risk reduction and prevention propose that person washing their hands with detergent to restrict the spread of virus infection and cut down the risk of unhealthiness. Due to the lack of soap and water, the CDC recommends that people employ alcohol-based (at least $60 \%$ ) hand sanitizer. According to scientific research by the world health organization (WHO), in the context of this coronavirus pandemic, hygienic as well as physical distancing are the best methods to protect ourselves and every person around us from COVID-19 infection. The virus is spread by people with this disease, and it can also be spread out via contact with sick people. We cannot treat the coronavirus with complete caution. Hence, good hand sanitization may be the ultimate obstacle between us and the virus. The WHO endorses using alcohol-based hand sanitizer to eliminate the new COVID-19 virus. Alcohol-based hand sanitizer can prevent microbial proteins (together with bacteria and certain viruses) from operating usually. Hand sanitizer needs to comprehend ethanol, isopropanol, npropanol, or a mixture of the alcohols. All of those can appropriately refuse to accept infections such as the new coronavirus. In that serious COVID-19 situation, the inquiry for hand sanitizer seems to have exaggerated. Due to exaggerated demand, it is difficult to obtain fine quality as well as multipurpose sanitizers in the overall market. The rise in demand also led to inferior sanitizers coming into the market. Akram et al. [55] utilized the GFFYWA operator for 


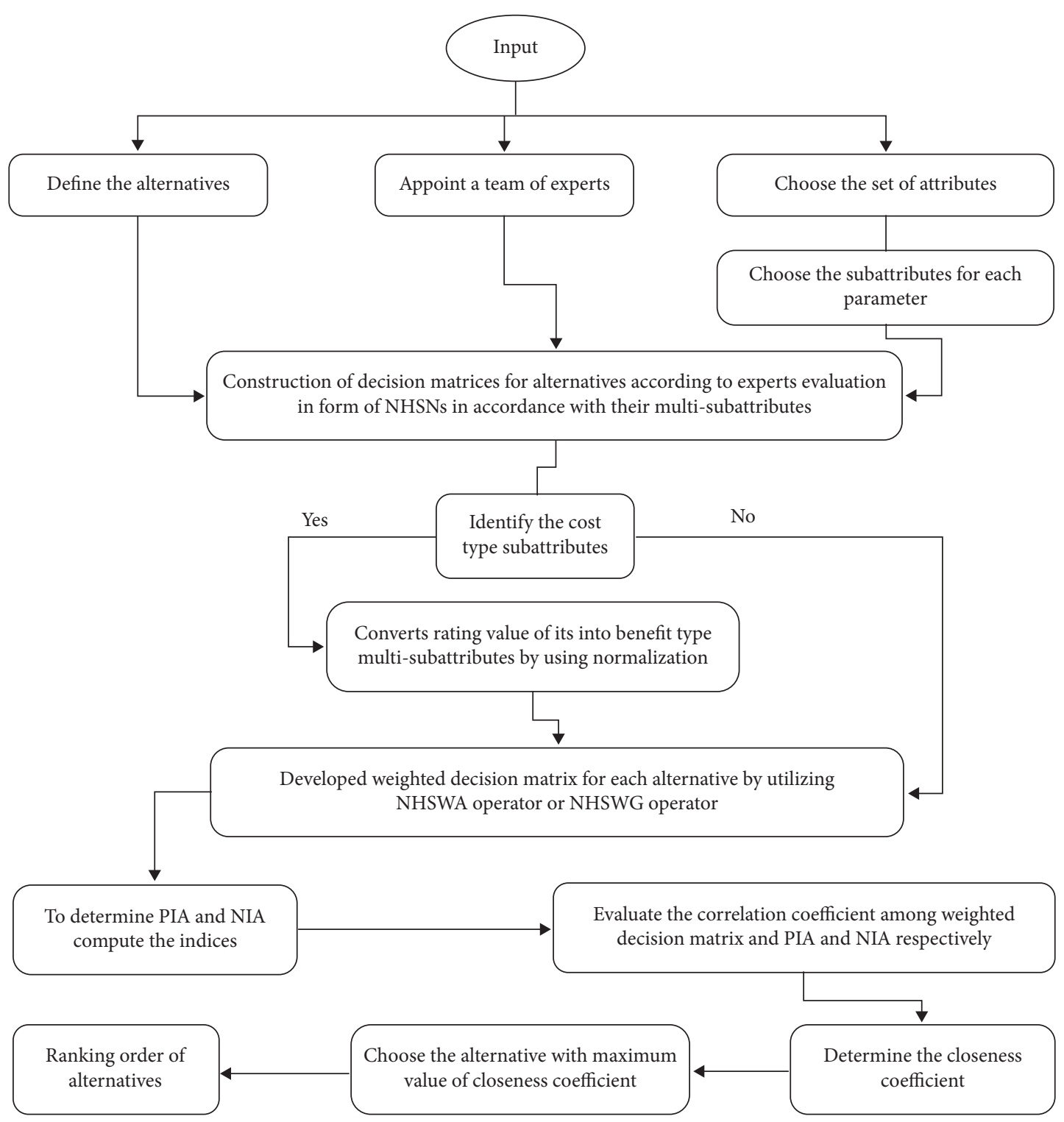

FIgURE 1: Graphical representation of developed TOPSIS technique for NHSS.

the selection of an effective hand sanitizer. The most important determinant of the current application is to reduce the spread of coronavirus by utilizing the TOPSIS technique under the NHSS scenario.

4.3. Numerical Example. Let $\mathscr{X}=\left\{\mathscr{X}_{1}, \mathscr{X}_{2}, \mathscr{X}_{3}, \mathscr{X}_{4}\right\}$ be a collection of four hand sanitizers (alternatives) such as $X_{1}=$ Megababe Squeaky Clean Sanitizer, $X_{2}=$ Purell Ad vanced Sanitizer Gel, $\mathscr{X}_{3}=$ Touchland power mist citrus, and $X_{4}=$ Lemon sanitizer spray. A team of four medical experts such as $\mathbb{Q}=\left\{Q^{1}, Q^{2}, Q^{3}, Q^{4}\right\}$ having weight vector
$(.1, .25, .35, .3)^{T}$ for the selection of an appropriate alternative (sanitizer), which is most helpful to protect the COVID-19 effects, is hired. The group of medical experts chooses the set of attributes for the selection of an effective sanitizer such as $\mathbb{Q}=\left\{\ell_{1}=\right.$ alcohol ratio, $\ell_{2}=$ ingredients, $\ell_{3}=$ Quality $\}$ with their corresponding subattribute: alcohol ratio $=\ell_{1}=\left\{d_{11}=\right.$ less than $40 \%, \mathrm{~d}_{12}=$ more than $\left.60 \%\right\}$, ingredients $=\ell_{2}=\quad\left\{d_{21}=\right.$ hydrogen peroxide, $d_{22}=$ glycerol $\}$, and quality $=\ell_{3}=\left\{d_{31}=\right.$ effectiveness $\}$. Let $\mathfrak{L}=\ell_{1} \times \ell_{2} \times \ell_{3}$ show the multi-subattributes:

$$
\begin{aligned}
\mathfrak{L}^{\prime} & =\ell_{1} \times \ell_{2} \times \ell_{3}=\left\{d_{11}, d_{12}\right\} \times\left\{d_{21}, d_{22}\right\} \times\left\{d_{31}\right\} \\
& =\left\{\left(d_{11}, d_{21}, d_{31}\right),\left(d_{11}, d_{22}, d_{31}\right),\left(d_{12}, d_{21}, d_{31}\right),\left(d_{12}, d_{22}, d_{31}\right)\right\} .
\end{aligned}
$$


TABle 1: Decision matrix for alternative $\mathscr{X}_{1}$.

\begin{tabular}{lcccr}
\hline $\mathscr{X}_{1}$ & $\check{\mathbf{d}}_{1}$ & $\check{\mathbf{d}}_{2}$ & $\check{\mathbf{d}}_{3}$ & \multicolumn{2}{c}{} \\
\hline $\mathscr{Q}^{1}$ & $(0.9,0.2,0.1)$ & $(0.3,0.3,0.7)$ & $(0.6,0.4,0.2)$ & $(0.7,0.1,0.3)$ \\
$\mathscr{Q}^{2}$ & $(0.8,0.3,0.2)$ & $(0.6,0.2,0.6)$ & $(0.8,0.3,0.1)$ & $(0.2,0.6,0.8)$ \\
$\mathscr{Q}^{3}$ & $(0.6,0.1,0.3)$ & $(0.6,0.1,0.3)$ & $(0.8,0.2,0.1)$ & $(0.6,0.3,0.4)$ \\
$\mathscr{Q}^{4}$ & $(0.9,0.1,0.1)$ & $(0.9,0.1,0.1)$ & $(0.8,0.1,0.1)$ & $(0.9,0.1,0.2)$ \\
\hline
\end{tabular}

TAвLE 2: Decision matrix for alternative $\mathscr{X}_{2}$.

\begin{tabular}{lcccc}
\hline $\mathscr{X}_{2}$ & $\check{\mathbf{d}}_{1}$ & $\check{\mathbf{d}}_{2}$ & $\check{\mathbf{d}}_{3}$ & $\check{\mathbf{d}}_{4}$ \\
\hline $\mathscr{Q}^{1}$ & $(0.3,0.3,0.7)$ & $(0.9,0.2,0.1)$ & $(0.6,0.1,0.3)$ & $(0.3,0.6,0.2)$ \\
$\mathscr{Q}^{2}$ & $(0.8,0.2,0.1)$ & $(0.8,0.3,0.2)$ & $(0.9,0.1,0.1)$ & $(0.8,0.3,0.1)$ \\
$\mathscr{Q}^{3}$ & $(0.6,0.3,0.4)$ & $(0.8,0.1,0.2)$ & $(0.9,0.1,0.1)$ & $(0.2,0.3,0.8)$ \\
$\mathscr{Q}^{4}$ & $(0.9,0.1,0.2)$ & $(0.8,0.1,0.1)$ & $(0.7,0.1,0.3)$ & $(0.6,0.3,0.4)$ \\
\hline
\end{tabular}

TABle 3: Decision matrix for alternative $\mathscr{X}_{3}$.

\begin{tabular}{lcccr}
\hline $\boldsymbol{X}_{3}$ & $\check{\mathbf{d}}_{1}$ & $\check{\mathbf{d}}_{2}$ & $\check{\mathbf{d}}_{3}$ & $\check{\mathbf{d}}_{4}$ \\
\hline $\mathbb{Q}^{1}$ & $(0.6,0.3,0.4)$ & $(0.2,0.3,0.8)$ & $(0.3,0.6,0.2)$ & $(0.3,0.6,0.2)$ \\
$\mathscr{Q}^{2}$ & $(0.9,0.1,0.1)$ & $(0.9,0.1,0.1)$ & $(0.9,0.1,0.1)$ & $(0.8,0.3,0.2)$ \\
$\widehat{Q}^{3}$ & $(0.8,0.3,0.2)$ & $(0.9,0.2,0.1)$ & $(0.9,0.1,0.1)$ & $(0.2,0.3,0.8)$ \\
$\mathscr{Q}^{4}$ & $(0.3,0.3,0.7)$ & $(0.9,0.1,0.2)$ & $(0.7,0.1,0.3)$ & $(0.6,0.3,0.4)$ \\
\hline
\end{tabular}

TABLE 4: Decision matrix for alternative $\mathscr{X}_{4}$.

\begin{tabular}{lcccc}
\hline $\boldsymbol{X}_{4}$ & $\breve{\mathbf{d}}_{1}$ & $\breve{\mathbf{d}}_{2}$ & $\breve{\mathbf{d}}_{3}$ & $\breve{\mathbf{d}}_{4}$ \\
\hline $\mathbb{Q}^{1}$ & $(0.9,0.1,0.1)$ & $(0.9,0.1,0.2)$ & $(0.8,0.2,0.1)$ & $(0.3,0.6,0.2)$ \\
$\mathscr{Q}^{2}$ & $(0.8,0.2,0.1)$ & $(0.8,0.2,0.1)$ & $(0.6,0.3,0.4)$ & $(0.8,0.3,0.2)$ \\
$\mathbb{Q}^{3}$ & $(0.8,0.1,0.1)$ & $(0.8,0.1,0.2)$ & $(0.9,0.1,0.1)$ & $(0.3,0.6,0.2)$ \\
$\mathscr{Q}^{4}$ & $(0.9,0.1,0.2)$ & $(0.3,0.3,0.7)$ & $(0.8,0.3,0.2)$ & $(0.9,0.1,0.1)$ \\
\hline
\end{tabular}

TABLE 5: Weighted decision matrix $\bar{X}_{1}$.

\begin{tabular}{lcccc}
\hline$\overline{\boldsymbol{X}}_{1}$ & $\check{\mathbf{d}}_{1}$ & $\check{\mathbf{d}}_{2}$ & $\check{\mathbf{d}}_{3}$ & $\check{\mathbf{d}}_{4}$ \\
\hline $\boldsymbol{Q}^{1}$ & $(0.7354,0.9835,0.5021)$ & $(0.5691,0.9778,0.9576)$ & $(0.6791,0.9762,0.8491)$ & $(0.6694,0.9848,0.8491)$ \\
$\widetilde{Q}^{2}$ & $(0.7200,0.9872,0.9009)$ & $(0.7038,0.9728,0.9413)$ & $(0.7955,0.9729,0.5476)$ & $(0.9009,0.9301,0.9835)$ \\
$\mathbb{Q}^{3}$ & $(0.7443,0.9438,0.4959)$ & $(0.6422,0.9513,0.9225)$ & $(0.7270,0.9798,0.8213)$ & $(0.7386,0.9465,0.9622)$ \\
$\mathscr{Q}^{4}$ & $(0.6328,0.9891,0.4784)$ & $(0.5034,0.9786,0.9888)$ & $(0.6829,0.9909,0.7866)$ & $(0.6377,0.9863,0.9945)$ \\
\hline
\end{tabular}

TABle 6: Weighted decision matrix $\overline{\mathscr{X}}_{2}$.

\begin{tabular}{lcccc}
$\overline{\boldsymbol{X}}_{2}$ & $\check{\mathbf{d}}_{1}$ & $\check{\mathbf{d}}_{2}$ & $\check{\mathbf{d}}_{3}$ & $\check{\mathbf{d}}_{4}$ \\
\hline $\mathbb{Q}^{1}$ & $(0.6867,0.9878,0.8073)$ & $(0.6178,0.9676,0.9889)$ & $(0.7920,0.9862,0.7920)$ & $(0.6694,0.9794,0.9862)$ \\
$\widetilde{Q}^{2}$ & $(0.7599,0.9754,0.8033)$ & $(0.6109,0.9517,0.9915)$ & $(0.7068,0.9529,0.7068)$ & $(0.9009,0.9301,0.9933)$ \\
$\mathscr{Q}^{3}$ & $(0.7849,0.9513,0.7749)$ & $(0.7379,0.9513,0.9498)$ & $(0.7819,0.9911,0.9083)$ & $(0.7386,0.9465,0.9377)$ \\
$\mathscr{Q}^{4}$ & $(0.7229,0.9891,0.7866)$ & $(0.4402,0.9908,0.9959)$ & $(0.6829,0.9880,0.7631)$ & $(0.5644,0.9821,0.9931)$ \\
\hline
\end{tabular}


Table 7: Weighted decision matrix $\overline{\mathscr{X}}_{3}$.

\begin{tabular}{lcccc}
\hline$\overline{\mathscr{X}}_{3}$ & $\check{\mathbf{d}}_{1}$ & $\check{\mathbf{d}}_{2}$ & $\check{\mathbf{d}}_{3}$ & $\check{\mathbf{d}}_{4}$ \\
\hline $\mathscr{Q}^{1}$ & $(0.7559,0.9835,0.8551)$ & $(0.6178,0.9754,0.9909)$ & $(0.7920,0.9862,0.5987)$ & $(0.6694,0.9729,0.9699)$ \\
$\mathscr{Q}^{2}$ & $(0.7599,0.9754,0.9070)$ & $(0.6109,0.9517,0.9893)$ & $(0.7068,0.9529,0.4661)$ & $(0.9009,0.9301,0.9236)$ \\
$\mathscr{Q}^{3}$ & $(0.8678,0.9257,0.8527)$ & $(0.6955,0.9362,0.9683)$ & $(0.9083,0.9529,0.6897)$ & $(0.7386,0.9465,0.9408)$ \\
$\mathscr{Q}^{4}$ & $(0.7229,0.9891,0.8457)$ & $(0.5034,0.9786,0.9974)$ & $(0.7408,0.9949,0.6195)$ & $(0.6049,0.9863,0.9919)$ \\
\hline
\end{tabular}

TABLE 8: Weighted decision matrix $\overline{\mathscr{X}}_{4}$.

\begin{tabular}{lcccc}
\hline$\overline{\mathscr{X}}_{4}$ & $\check{\mathbf{d}}_{1}$ & $\check{\mathbf{d}}_{2}$ & $\check{\mathbf{d}}_{3}$ & $\check{\mathbf{d}}_{4}$ \\
\hline $\mathscr{Q}^{1}$ & $(0.6867,0.9915,0.8241)$ & $(0.6572,0.9754,0.9840)$ & $(0.7311,0.9818,0.5021)$ & $(0.7048,0.9645,0.9605)$ \\
$\mathscr{Q}^{2}$ & $(0.7599,0.9754,0.9070)$ & $(0.6109,0.9517,0.9821)$ & $(0.9085,0.9333,0.6509)$ & $(0.7781,0.9301,0.9553)$ \\
$\mathscr{Q}^{3}$ & $(0.8678,0.9438,0.8527)$ & $(0.7379,0.9513,0.9683)$ & $(0.8886,0.9377,0.6415)$ & $(0.8376,0.9465,0.9225)$ \\
$\mathscr{Q}^{4}$ & $(0.7229,0.9891,0.8457)$ & $(0.4402,0.9786,0.9965)$ & $(0.7152,0.9931,0.6575)$ & $(0.6667,0.9821,0.9506)$ \\
\hline
\end{tabular}

$\mathfrak{Q}^{\prime}=\left\{\check{d}_{1}, \check{d}_{2}, \check{d}_{3}, \check{d}_{4}\right\} \quad$ with weights $(0.2,0.1,0.4,0.3)^{T}$. Each expert will evaluate the ratings of hand sanitizer in the form of NHSNs for each subattribute.

\subsubsection{Selection of an Effective Hand Sanitizer by Using the TOPSIS Method}

Step 1: decision makers will investigate the situation in the case of NHSNs, and there are only four alternatives. The subattributes of the considered attributes, along with a summary of their scores are given in Tables 1-4.
Step 2: there is no need for normalization since all attributes are identical.

Step 3: weighted decision matrix for each alternative $\overline{\mathscr{X}}_{z}=\left(\overline{\mathscr{L}}_{i j}^{(z)}\right)_{n \times \partial}$ is developed by using equation (30). The values of the weighted decision matrices for each alternative are given in Tables 5-8.

Step 4: by utilizing equations (35) and (36), compute the PIA and NIA such as follows:

$$
\begin{array}{r}
\mathscr{L}^{+}=\left(\begin{array}{llll}
(0.6867,0.9878,0.8073) & (0.6178,0.9676,0.9889) & (0.7311,0.9818,0.5021) & (0.6694,0.9729,0.9699) \\
(0.7599,0.9754,0.9070) & (0.6109,0.9517,0.9915) & (0.7068,0.9529,0.7068) & (0.9009,0.9301,0.9835) \\
(0.7849,0.9513,0.7749) & (0.7379,0.9513,0.9498) & (0.7270,0.9798,0.8213) & (0.8376,0.9465,0.9225) \\
(0.7229,0.9891,0.8457) & (0.5034,0.9786,0.9974) & (0.6829,0.9909,0.7866) & (0.6049,0.9863,0.9919)
\end{array}\right), \\
\mathscr{L}^{-}=\left(\begin{array}{llll}
(0.7354,0.9835,0.5021) & (0.6572,0.9754,0.9840) & (0.6791,0.9762,0.8491) & (0.7048,0.9645,0.9605) \\
(0.7200,0.9872,0.9009) & (0.7038,0.9728,0.9413) & (0.7955,0.9729,0.5476) & (0.9009,0.9301,0.9236) \\
(0.8678,0.9438,0.8527) & (0.7379,0.9513,0.9683) & (0.9083,0.9529,0.6897) & (0.7386,0.9465,0.9622) \\
(0.6328,0.9891,0.4784) & (0.4402,0.9908,0.9959) & (0.7152,0.9931,0.6575) & (0.6667,0.9821,0.9506)
\end{array}\right) .
\end{array}
$$

Step 5: by using equation (37), compute the CC such as $p^{(1)}=0.99915, \quad p^{(2)}=0.99811, \quad p^{(3)}=0.99746, \quad$ and $p^{(4)}=0.99787$.

Step 6: by using equation (38), compute the CC such as $q^{(1)}=0.99742, \quad q^{(2)}=0.99806, \quad q^{(3)}=0.99870, \quad$ and $q^{(4)}=0.99870$.

Step 7: for the alternatives ranking, compute the closeness coefficient by using equation (39):

$$
\begin{aligned}
& \mathscr{R}^{(1)}=0.75219, \\
& \mathscr{R}^{(2)}=0.50653, \\
& \mathscr{R}^{(3)}=0.33854, \\
& \mathscr{R}^{(4)}=0.37901 .
\end{aligned}
$$

Step 8: pick the alternate with the largest closeness coefficient $\mathscr{R}^{(1)}=0.75219$, so $\mathscr{X}^{(1)}$ will be the best alternative.

Step 9: analyze the ranking: $\mathscr{R}^{(1)}>\mathscr{R}^{(2)}>$ $\mathscr{R}^{(4)}>\mathscr{R}^{(3)}$, so the ranking of the alternatives is $\mathscr{X}^{(1)}>\mathscr{X}^{(2)}>\mathscr{X}^{(4)}>\mathscr{X}^{(3)}$.

\section{Discussion and Comparative Analysis}

In the subsequent section, we are going to talk over the effectiveness, simplicity, and tractability along with the benefits of the planned approach. We also performed a brief comparison of the undermentioned: the planned approach along with some existing techniques. 
TABLE 9: Comparison between NHSS and some existing techniques.

\begin{tabular}{lccccccc}
\hline & Set & Truthiness & indeterminacy & Falsity & Parametrization & Attributes & Subattributes \\
\hline Zadeh [1] & FS & $\checkmark$ & $\times$ & $\times$ & $\times$ & $\checkmark$ & $\times$ \\
Atanassov [2] & IFS & $\checkmark$ & $\times$ & $\checkmark$ & $\times$ & $\checkmark$ & $\checkmark$ \\
Maji et al. [21] & FSS & $\checkmark$ & $\times$ & $\checkmark$ & $\checkmark$ & $\checkmark$ \\
Maji et al. [22] & IFSS & $\checkmark$ & $\checkmark$ & $\checkmark$ & $\checkmark$ & $\checkmark$ \\
Peng et al. [26] & PFSS & $\checkmark$ & $\checkmark$ & $\checkmark$ & $\checkmark$ & $\checkmark$ \\
Zulqarnain et al. [46] & IFHSS & $\checkmark$ & $\checkmark$ & $\checkmark$ & $\checkmark$ \\
Zulqarnain et al. [48] & PFHSS & $\checkmark$ & $\checkmark$ & $\checkmark$ & $\checkmark$ \\
Proposed approach & NHSS & $\checkmark$ & $\checkmark$ & $\checkmark$ & $\checkmark$ \\
\hline
\end{tabular}

5.1. Comparative Analysis. Through the current exploration along with comparative analysis in Table 9, it can be concluded that the results acquired by the proposed approach overlap with the accessible methodologies. However, in connection with accessible decision-making techniques, the main benefit of the proposed method is that it comprises additional information utilizing the membership, nonmembership, and indeterminacy of subattributes of considered attributes to address uncertainty in data. Among them, the information related to the object can be interpreted more accurately and objectively. It is also a useful tool for solving inaccurate and imprecise data in the DM process. Also, the calculation process of the developed method is different from the available techniques. This is due to the determination of PIA and NIA. In preceding techniques, PIA and NIA were conceptualized according to a given distance and similarity measures. Therefore, the inspiration for the score value corresponding to each parameter will not affect other parameters, so predictable information loss will occur in the process. On the contrary, in the proposed method, PIA and NIA are calculated based on the inspiration of the maximum CC on a given substitution level, so there is no serious loss of information in the process. After all, the intensity of each ideal correlation measure is obtained from the assessment explanations, so to find the superlative result compute the correlation between them. The benefit of the planned TOPSIS method along with related measures over existing methods is that it notices not only the degree of discrimination but also the degree of similarity between observations so that avoiding decisions based on negative reasons. Therefore, it is a suitable tool to combine inaccurate as well as uncertain info in the DM process. Hence, our suggested approach is effective, flexible, simple, and in advance of distinctive hybrid structures of FS.

\section{Discussion}

By utilizing the methodology of Zadeh [1], we can process the $\mathrm{MD}$ of the alternative, but the FS has no information about the NMD of the considered parameters. Atanassov [2] deals with the uncertainty by using MD and NMD. However, these theories cannot deal with the situation when the sum of MD and NMD exceeds one; on the other hand, our presented notion competently deals with such difficulties. Meanwhile, these theories have no information about the parametrization of the attributes. To overcome such difficulties, Maji et al. [21] presented the notion of FSS to deal with the parametrization of the objects which contains uncertainty by considering the MD of the attributes. However, the presented FSS provides no information about the NMD of the object. To overcome the presented drawback, Maji et al. [22] offered the concept of IFSS, the presented notion handles the uncertain object more accurately by utilizing the MD and NMD of the attributes with their parametrization, and the sum of MD and NMD does not exceed 1. To handle this scenario, Peng et al. [26] proposed the notion of PFSS by modifying the condition $\mathrm{MD}+\mathrm{NMD} \leq 1$ to $\mathrm{MD}^{2}+\mathrm{NMD}^{2} \leq 1$ with their parametrization. However, all the abovementioned studies have no information about the subattributes of the considered attributes; when attributes have their corresponding subattributes, then all abovementioned theories fail to handle the situation. Zulqarnain et al. [46] presented the TOPSIS technique under the IFHSS environment to deal with uncertain problems by using MD and NMD in which the sum of subattributes of the considered parameters cannot exceed one. However, IFHSS cannot provide any information on the indeterminacy of the subattribute of the considered attribute. Zulqarnain et al. [48] proposed the more generalized notion of PFHSS comparative to IFHSS. All existing hybrid structures of FS cannot handle the indeterminacy of subattributes of considered $n$-tuple attributes. To overcome the above limitations, we prolonged the IFHSS to NHSS. Instead, the method we developed is a progressive approach that can handle alternatives with multiple subattribute info by using truthiness, indeterminacy, and falsity object with the following condition $0 \leq \leq \mathscr{T}_{\mathscr{F}(\breve{a})}(v)+\mathscr{I}_{\mathscr{F}(\breve{a})}(v)+$ $\mathfrak{C}_{\mathscr{F}(\breve{a})}(v) \leq 3$. Consequently, relying on the obtained results, it can be confidently concluded that the proposed methodology indicates higher stability and usability for decision makers in the DM procedure.

\section{Conclusion}

This study focuses on NHSS to solve the problem of insufficient data, ambiguity, and inconsistency by considering the degree of truthiness, falsity, and indeterminacy on the $n$ tuple subattributes of the considered attributes. This research puts forward the new concepts and properties of CC and WCC in the NHSS environs. Based on the proposed correlation measure, by considering the $n$-tuple subattributes as well as decision makers, the TOPSIS method is extended in the neutrosophic hypersoft environment. To get the overall top-ranking of possible choices, we characterized 
the closeness coefficient under the constructed TOPSIS approach. We have also defined NHSWA and NHSWG operators and proposed the DM strategy according to the developed TOPSIS technique. To resolve the MCGDM problem, a numerical illustration has been described by utilizing the recommended TOPSIS approach for the selection of more suitable hand sanitizer (alternative) during the COVID-19 pandemic. Moreover, a comparison has been conducted for the justification along with effectivity of the approach. Consequently, relying upon the obtained outcomes, it can be confidently concluded that the proposed methodology indicates greater constancy as well as usability for decision makers in the DM process. Future research will surely concentrate on presenting several other operators to solve decision-making complications utilizing NHSS. Many other structures such as topological, algebraic, and ordered structures can be developed and discussed under-considered environment. The suggested idea can be applied to quite a lot of issues in real life, including the medical profession, robotics, artificial intelligence, pattern recognition, and economics. We are sure this article will open new vistas for investigators in this field.

\section{Data Availability}

No data were used to support the findings of the study.

\section{Conflicts of Interest}

The authors declare that they have no conflicts of interest.

\section{Authors' Contributions}

Abdul Samad and Rana Muhammad Zulqarnain contributed equally to this work.

\section{Acknowledgments}

Rifaqat Ali would like to express his gratitude to Deanship of Scientific Research at King Khalid University, Saudi Arabia, for providing funding research groups under the research Grant no. R.G.P. 2/71/41.

\section{References}

[1] L. A. Zadeh, "Fuzzy sets," Information and Control, vol. 8, no. 3, pp. 338-353, 1965.

[2] K. T. Atanassov, "Intuitionistic fuzzy sets," Fuzzy Sets and Systems, vol. 20, no. 1, pp. 87-96, 1986.

[3] R. R. Yager, "Pythagorean fuzzy subsets," Proceedings of the Joint IFSA World Congress and NAFIPS Annual Meeting, vol. 20, 2013.

[4] R. R. Yager, "Pythagorean membership grades in multicriteria decision making," IEEE Transactions on Fuzzy Systems, vol. 22, no. 4, pp. 958-965, 2014.

[5] X. Zhang and Z. Xu, "Extension of TOPSIS to multiple criteria decision making with pythagorean fuzzy sets," International Journal of Intelligent Systems, vol. 29, no. 12, pp. 1061-1078, 2014.

[6] G. Wei and M. Lu, "Pythagorean fuzzy power aggregation operators in multiple attribute decision making,"
International Journal of Intelligent Systems, vol. 33, no. 1, pp. 169-186, 2018.

[7] L. Wang and N. Li, "Pythagorean fuzzy interaction power bonferroni mean aggregation operators in multiple attribute decision making," International Journal of Intelligent Systems, vol. 35, no. 1, pp. 150-183, 2020.

[8] H. Gao, M. Lu, and G. Wei, "Some novel pythagorean fuzzy interaction aggregation operators in multiple attribute decision making," Fundamenta Informaticae, vol. 159, no. 4, pp. 385-428, 2018.

[9] G. Wei, "Pythagorean fuzzy interaction aggregation operators and their application to multiple attribute decision making," Journal of Intelligent \& Fuzzy Systems, vol. 33, no. 4, pp. 2119-2132, 2017.

[10] X. Zhang, "A novel approach based on similarity measure for pythagorean fuzzy multiple criteria group decision making," International Journal of Intelligent Systems, vol. 31, no. 6, pp. 593-611, 2016.

[11] L. Wang, H. Garg, and N. Li, "Pythagorean fuzzy interactive hamacher power aggregation operators for assessment of express service quality with entropy weight," Soft Computing, vol. 31, pp. 1-21, 2020.

[12] L. Wang and N. Li, "Continuous interval-valued Pythagorean fuzzy aggregation operators for multiple attribute group decision making," Journal of Intelligent \& Fuzzy Systems, vol. 36, no. 6, p. 6245, 2019.

[13] X. Peng and H. Yuan, "Fundamental properties of pythagorean fuzzy aggregation operators," Fundamenta Informaticae, vol. 147, no. 4, pp. 415-446, 2016.

[14] X. Peng and Y. Yang, "Some results for pythagorean fuzzy sets," International Journal of Intelligent Systems, vol. 30, no. 11, pp. 1133-1160, 2015.

[15] H. Garg, "New logarithmic operational laws and their aggregation operators for pythagorean fuzzy set and their applications," International Journal of Intelligent Systems, vol. 34, no. 1, p. 82, 2019.

[16] R. Arora and H. Garg, "Group decision-making method based on prioritized linguistic intuitionistic fuzzy aggregation operators and its fundamental properties," Computational and Applied Mathematics, vol. 38, no. 2, pp. 1-36, 2019.

[17] Z. Ma and Z. Xu, "Symmetric pythagorean fuzzy weighted geometric/averaging operators and their application in multicriteria decision-making problems," International Journal of Intelligent Systems, vol. 31, no. 12, pp. 1198-1219, 2016.

[18] D. Molodtsov, "Soft set theory-First results," Computers \& Mathematics with Applications, vol. 37, no. 4-5, pp. 19-31, 1999.

[19] P. K. Maji, R. Biswas, and A. R. Roy, "Soft set theory," Computers \& Mathematics with Applications, vol. 45, no. 4-5, pp. 555-562, 2003.

[20] P. K. Maji, A. R. Roy, and R. Biswas, "An application of soft sets in a decision making problem," Computers \& Mathematics with Applications, vol. 44, no. 8-9, pp. 1077-1083, 2002.

[21] P. K. Maji, R. Biswas, and A. R. Roy, "Fuzzy soft sets," Journal of Fuzzy Mathematics, vol. 9, pp. 589-602, 2001.

[22] P. K. Maji, R. Biswas, and A. R. Roy, "Intuitionistic fuzzy soft sets," Journal of Fuzzy Mathematics, vol. 9, pp. 677-692, 2001.

[23] H. Garg and R. Arora, "Generalized and group-based generalized intuitionistic fuzzy soft sets with applications in decision-making," Applied Intelligence, vol. 48, no. 2, pp. 343-356, 2018.

[24] H. Garg and R. Arora, "TOPSIS method based on correlation coefficient for solving decision-making problems with 
intuitionistic fuzzy soft set information," AIMS Mathematics, vol. 5, no. 4, pp. 2944-2966, 2020.

[25] R. M. Arora, X. L. Xin, M. Saqlain, and W. A. Khan, "TOPSIS method based on the correlation coefficient of interval-valued intuitionistic fuzzy soft sets and aggregation operators with their application in decision-making," Journal of Mathematics, vol. 2021, pp. 1-16, 2021.

[26] X. Peng, Y. Yang, and J. Song, "Pythagoren fuzzy soft set and its application," Computer Engineering, vol. 41, no. 7, pp. 224-229, 2015.

[27] T. M. Athira, S. J. John, and H. Garg, "A novel entropy measure of pythagorean fuzzy soft sets," AIMS Mathematics, vol. 5, no. 2, pp. 1050-1061, 2020.

[28] T. M. Athira, S. J. John, and H. Garg, "Entropy and distance measures of pythagorean fuzzy soft sets and their applications," Journal of Intelligent \& Fuzzy Systems, vol. 37, no. 3, pp. 4071-4084, 2019.

[29] R. M. Zulqarnain, X. L. Xin, H. Garg, and W. A. Khan, "Aggregation operators of pythagorean fuzzy soft sets with their application for green supplier chain management," Journal of Intelligent \& Fuzzy Systems, vol. 40, no. 3, p. 5545, 2021.

[30] M. Riaz, K. Naeem, and D. Afzal, "Pythagorean m-polar fuzzy soft sets with TOPSIS method for MCGDM," Punjab University Journal of Mathematics, vol. 52, no. 3, pp. 21-46, 2020.

[31] M. Riaz, N. Khalid, and D. Afzal, "A similarity measure under pythagorean fuzzy soft environment with applications," Computational and Applied Mathematics, vol. 39, no. 4, pp. 1-17, 2020.

[32] Q. Han, W. Li, Y. Song, T. Zhang, and R. Wang, "A new method for MAGDM based on improved TOPSIS and a novel pythagorean fuzzy soft entropy," Symmetry, vol. 11, no. 7, p. 905, 2019.

[33] R. M. Zulqarnain, X. L. Xin, I. Siddique, W. Asghar Khan, and M. A. Yousif, "TOPSIS method based on correlation coefficient under pythagorean fuzzy soft environment and its application towards green supply chain management," Sustainability, vol. 13, no. 4, p. 1642, 2021.

[34] F. Smarandache, "Neutrosophy: neutrosophic probability, set, and logic: analytic synthesis \& synthetic analysis," 1998.

[35] P. K. Maji, "Neutrosophic soft set," Annals of Fuzzy Mathematics and Informatics, vol. 5, no. 1, pp. 157-168, 2013.

[36] F. Karaaslan, "Possibility neutrosophic soft sets and PNSdecision making method," Applied Soft Computing Journal, vol. 54, pp. 403-414, 2016.

[37] S. Broumi, "Generalized neutrosophic soft set," International Journal of Computer Science, Engineering and Information Technology, vol. 3, no. 2, pp. 17-30, 2013.

[38] I. Deli and Y. Şubaş, "A ranking method of single valued neutrosophic numbers and its applications to multi-attribute decision making problems," International Journal of Machine Learning and Cybernetics, vol. 8, no. 4, pp. 1309-1322, 2017.

[39] H. Wang, F. Smarandache, and Y. Zhang, "Single valued neutrosophic sets," International Journal of Machine Learning and Cybernetics, vol. 42, pp. 386-394, 2013.

[40] J. Ye, "A multicriteria decision-making method using aggregation operators for simplified neutrosophic sets," Journal of Intelligent \& Fuzzy Systems, vol. 26, no. 5, pp. 2459-2466, 2014.

[41] M. R. Hashmi, M. Riaz, and F. Smarandache, " $m$-Polar neutrosophic topology with applications to multi-criteria decision-making in medical diagnosis and clustering analysis," International Journal of Fuzzy Systems, vol. 22, no. 1, pp. 273-292, 2020.
[42] R. M. Zulqarnain, X. L. Xin, M. Saeed, F. Smarandache, and N. Ahmad, "Generalized neutrosophic TOPSIS to solve multicriteria decision-making problems," Neutrosophic Sets and Systems, vol. 38, pp. 276-292, 2020.

[43] F. Smarandache, "Extension of soft set to hypersoft set, and then to plithogenic hypersoft set," Neutrosophic Sets and Systems, vol. 22, pp. 168-170, 2018.

[44] S. Rana, M. Qayyum, M. Saeed, and F. Smarandache, "Plithogenic fuzzy whole hypersoft set: construction of operators and their application in frequency matrix multi attribute decision making technique," Neutrosophic Sets and Systems, vol. 28, pp. 34-50, 2019.

[45] R. M. Zulqarnain, X. L. Xin, M. Saqlain, and F. Smarandache, "Generalized aggregate operators on neutrosophic hypersoft set," Neutrosophic Sets and Systems, vol. 36, pp. 271-281, 2020.

[46] R. M. Zulqarnain, X. L. Xin, and M. Saeed, "Extension of TOPSIS method under intuitionistic fuzzy hypersoft environment based on correlation coefficient and aggregation operators to solve decision making problem," AIMS Mathematics, vol. 6, 2020.

[47] R. M. Zulqarnain, X. L. Xin, and M. Saeed, "A Development of Pythagorean fuzzy hypersoft set with basic operations and decision-making approach based on the correlation coefficient," Theory and Application of Hypersoft Set, vol. 6, 2021.

[48] R. M. Zulqarnain, X. L. Xin, and M. Saeed, "Development of topsis technique under pythagorean fuzzy hypersoft environment based on correlation coefficient and its application towards the selection of antivirus mask in covid-19 pandemic," Complexity, vol. 6, 2021.

[49] R. M. Zulqarnain, X. L. Xin, M. Saqlain, M. Saeed, F. Smarandache, and M. I. Ahamad, "Some fundamental operations on interval valued neutrosophic hypersoft set with their properties," Neutrosophic Sets, and Systems, vol. 40, pp. 134-148, 2021.

[50] R. M. Zulqarnain, X. L. Xin, B. Ali, S. Broumi, S. Abdal, and M. I. Ahamad, "Decision-making approach based on correlation coefficient with its properties under interval-valued neutrosophic hypersoft set environment," Neutrosophic Sets, and Systems, vol. 40, pp. 12-28, 2021.

[51] C. L. Hwang and K. Yoon, Multiple Attribute Decision Making Methods and Applications A State-of-the-Art Survey, SpringerVerlag Berlin Heidelberg, London, UK, 1981.

[52] P. P. Dixit, "Alcohol based hand sanitizers: assurance and apprehensions revisited," Research Journal of Pharmaceutical, Biological and Chemical Sciences, vol. 5, pp. 558-563, 2014.

[53] A. Kramer and S. A. Sattar, "Activity of a new hand disinfectant with reduced ethanol content: comparison with other alcohol-based formulations," Journal of Hospital Infection, vol. 62, no. 1, pp. 98-106, 2006.

[54] V. Döhner, T. J. Daha, H. Brug et al., "Systematic review of studies on compliance with hand hygiene guidelines in hospital care," Infection Control \& Hospital Epidemiology, vol. 31, no. 3, pp. 283-294, 2010.

[55] M. Akram, G. Shahzadi, and A. A. H. Ahmadini, "Decisionmaking framework for an effective sanitizer to reduce COVID-19 under fermatean fuzzy environment," Journal of Mathematics, vol. 3, 2020. 